\title{
Development of Two-Fluid Magnetohydrodynamics Model for Non-Equilibrium Anisotropic Plasma Flows
}

\author{
K. Miura* and C. P. T. Groth ${ }^{\dagger}$ \\ University of Toronto Institute for Aerospace Studies \\ 4925 Dufferin Street, Toronto, Ontario, M3H 5T6, Canada
}

\begin{abstract}
A multi-species magnetohydrodynamic (MHD) model based on an extended fluid dynamics description for each plasma species is proposed for the prediction of the flow and behavior of fully ionized non-equilibrium anisotropic plasmas. In particular, a two-fluid (ions and electrons) plasma model is described that makes use of a 10-moment or Gaussian anisotropic moment closure to model ion and electron species transport. The ion and electron moment equations are fully coupled to the complete set of Maxwell's equations which govern electromagnetic wave propagation within the plasma and a relaxation time approximation is used to model non-equilibrium Coulomb collisional processes between the ions and electrons. Unlike conventional MHD models, the proposed two-fluid model is capable of taking into account large temperature anisotropies and temperature differences between the electrons and ions, both of which can occur for low-density, high-temperature plasmas and/or strongly magnetized plasmas. A dispersion analysis of the proposed model is undertaken in order to examine further its mathematical properties and explore the nature of its solutions. The dispersive and hyperbolic nature of the equations and the disparate times scales or plasma frequencies of the solutions are revealed. The governing system of partial differential equations would appear to be well suited for solution by Godunov-type finite-volume methods provided that the disparate scales can be dealt with. A higherorder finite-volume method is developed here for the solution of the one-dimensional form of the two-fluid plasma model and dispersion analyses of the discretized equations indicates that a stable and practical scheme is possible if a fully implicit time marching scheme is adopted. The analysis also suggests mechanisms for preconditioning the system of equations to further reduce numerical stiffness, resulting in a plasma model that is more suitable for practical applications. Numerical results for several one-dimensional unsteady plasma flows are described and demonstrate the potential of the proposed multi-species plasma model for predicting non-equilibrium anisotropic plasma flows in engineering applications such as those encountered in electric space propulsion devices. The future extension of the model to partially ionized plasmas in two and three space dimensions is also discussed.
\end{abstract}

\section{Introduction}

The study and modelling of plasmas have many applications in the field of aerospace engineering. Applications ranging from deep space propulsion to high speed flight ${ }^{1,2}$ exist where plasma modelling can help in advanced design analysis, as well as in aiding in the actual manufacturing and material processes used to build these vehicles. Plasma modelling is particularly important for space Electric Propulsion (EP) systems. ${ }^{3-5}$ This class of space propulsion was made famous by the Deep Space 1 (DS1) probe but has been extensively researched for decades now. ${ }^{6}$

With the recent successes in space tested EP, a variety of new and old EP systems are now being considered. These include ion thruster designs similar to that of DS1, ${ }^{7}$ and the SMART-1, which uses a Hall thruster.$^{8-10}$ Other EP systems include the high powered arcjet thrusters, ${ }^{11}$ the high thrust VASIMR ${ }^{12}$ and low power electro-thermal thrusters. ${ }^{13}$ A good overview of EP technology can be found in the paper by Martinez-Sanchez and Pollard. ${ }^{3}$ As advanced EP systems become more and more common, designing

*PhD Candidate, Email: miura@utias.utoronto.ca, Student Member AIAA

$\dagger$ Associate Professor, Email: groth@utias.utoronto.ca, Senior Member AIAA 
and testing these systems requires equally advanced plasma modelling to simulate and predict the plasma transport phenomena within the propulsion system.

Collectively, the study of flows and interactions of plasmas with electromagnetic forces is known as magnetohydrodynamics (MHD). ${ }^{14-16}$ The modelling of plasmas can be considerably more complicated than regular fluid dynamic flows as there is the added complexity of the electro-magnetic forces (such as the Lorentz force) along with the widely varying physical characteristics of the plasma species (i.e., electrons and ions).

Current MHD models border on two extremes. On the one side, we have the 5-moment MHD model which assumes isotropic temperatures and pressures and does not differentiate between the electron and ion temperatures. ${ }^{14,15}$ This description is commonly known as the ideal MHD equations and is used in many applications, such as for the modelling of space plasmas. ${ }^{17,}{ }^{18}$ On the other end of the spectrum we have direct particle simulations. ${ }^{19,20}$ In direct particle simulations, individual particles and/or groupings of particles are tracked and their collisions are simulated. Obviously, the simplified, ideal MHD description is not always valid, especially for EP systems which often take advantage of the anisotropic nature of plasmas. ${ }^{12}$ Moreover, the direct particle simulations can require significant computational resources which can prohibit their application to practical problems. For example, near-equilibrium or high-density regimes are very challenging to treat accurately if the particle simulation model is used for the whole or even a part of the problem. ${ }^{21}$

Attempts have been made to extend the capabilities of the ideal MHD model, to include non-equilibrium effects without resorting to a direct particle simulation. One approach is to consider multispecies models such as the two-fluid model of Shumlak and Loverich, ${ }^{22}$ where separate 5 -moment equilibrium transport equations are used to represent the ions and electrons. The advantage of this model is the fact that each of the species and the electromagnetic (EM) fields are then represented by a complete set of partial differential equations, each of which can be treated numerically in different ways. This automatically allows for differences in ion and electron temperatures and velocities. The disadvantage is the inherent stiffness of the equations and the computational effort that can be required to solve the system of equations. The subject of this paper is to develop an extension of the two-fluid model of Shumlak and Loverich to include plasma species temperature anisotropies and interparticle collision effects. These important non-equilibrium plasma effects are incorporated by using a 10-moment Gaussian closure to model the non-equilibrium transport of the plasma species, and interparticle collision effects are modelled using a relaxation time approximation. At the same time, the model should be designed to be computationally tractable for performing plasma simulations of practical space propulsion devices. Numerical aspects of the proposed anisotropic plasma model are also examined.

\section{Multispecies Transport Equations}

A two-fluid plasma model based on the 10-moment Gaussian closure can be derived by considering the Boltzmann kinetic equation for species $s$ given by

$$
\frac{\partial \mathrm{F}_{s}\left(\mathbf{x}, \mathbf{v}_{s}, t\right)}{\partial t}+v_{s k} \frac{\partial \mathrm{F}_{s}\left(\mathbf{x}, \mathbf{v}_{s}, t\right)}{\partial x_{k}}+a_{s k} \frac{\partial \mathrm{F}_{s}\left(\mathbf{x}, \mathbf{v}_{s}, t\right)}{\partial v_{s k}}=\frac{\delta \mathrm{F}_{s}\left(\mathbf{x}, \mathbf{v}_{s}, t\right)}{\delta t}
$$

where $\mathrm{F}_{s}\left(\mathbf{x}, \mathbf{v}_{s}, t\right)$ is the distribution function, $\mathbf{x}$ is the position vector, $\mathbf{v}_{s}$ is the velocity vector for species $s, t$ is the time, and $a_{s k}$ is the acceleration of species $s$. The Boltzmann equation describes the time evolution of the distribution function. The term on the right hand side is the Boltzmann collision integral, and incorporates the effect of interparticle collisions. The transport equations of the 10-moment Gaussian closure can be derived by approximating the distribution function $\mathrm{F}_{s}\left(\mathbf{x}, \mathbf{v}_{s}, t\right)$ with the Gaussian distribution function and then taking appropriate velocity moments of the Boltzmann equation. ${ }^{23-25}$ The 10-moment Gaussian closure is ideal for the present work because of its inherent ability to model anisotropic pressures and temperatures. Furthermore, it has been recently applied successfully to a range of non-magnetized rarefied gaseous flows. ${ }^{26-28}$ In the Gaussian closure, the species phase space distribution function is approximated as follows:

$$
\mathcal{G}_{s}\left(\mathbf{x}, \mathbf{v}_{s}, t\right)=\frac{n_{s}(\mathbf{x}, t)}{(2 \pi)^{3 / 2}\left(\operatorname{det} \Theta_{s}\right)^{1 / 2}} e^{\left(-\frac{1}{2} \Theta_{s i j}^{-1} c_{s i} c_{s j}\right)}
$$

where $\Theta_{s i j}=P_{s i j} / \rho_{s}$. When applied to a two species plasma consisting of ions and electrons, and coupled with Maxwell's equations to prescribe the electromagnetic forces, the resulting complete set of governing 
equations can be expressed in weak conservation form as

$$
\frac{\partial \mathbf{U}}{\partial t}+\frac{\partial \mathbf{F}}{\partial x_{i}}=\mathbf{S}+\mathbf{S}^{c o l}
$$

with

$$
\mathbf{U}=\left(\begin{array}{c}
\mathbf{U}_{i o n} \\
\mathbf{U}_{e} \\
\mathbf{U}_{M}
\end{array}\right), \quad \mathbf{F}=\left(\begin{array}{c}
\mathbf{F}_{i o n} \\
\mathbf{F}_{e} \\
\mathbf{F}_{M}
\end{array}\right), \quad \mathbf{S}=\left(\begin{array}{c}
\mathbf{S}_{i o n} \\
\mathbf{S}_{e} \\
\mathbf{S}_{M}
\end{array}\right), \quad \mathbf{S}^{c o l}=\left(\begin{array}{c}
\mathbf{S}_{i o n}^{c o l} \\
\mathbf{S}_{e}^{c o l} \\
\mathbf{S}_{M}^{c o l}
\end{array}\right)
$$

where

$$
\begin{gathered}
\mathbf{U}_{s}=\left(\begin{array}{c}
\rho_{s} \\
\rho_{s} u_{s, k} \\
\rho_{s} u_{s, j} u_{s, k}+P_{s, j k}
\end{array}\right), \mathbf{F}_{s}=\left(\begin{array}{c}
\rho_{s} u_{s, i} \\
\rho_{s} u_{s, i} u_{s, k}+P_{s, i k} \\
\mathbf{S}_{s}=\left(\begin{array}{c}
\rho_{s, i} u_{s, j} u_{s, k}+P_{s, j k} u_{s, i}+ \\
+P_{s, i k} u_{s, j}+P_{s, i j} u_{s, k}
\end{array}\right) \\
q_{s} \frac{\rho_{s}}{m_{s}}\left(E_{k}+\epsilon_{k \alpha \gamma} u_{s \alpha} B_{\gamma}\right) \\
q_{s} \frac{\rho_{s}}{m_{s}}\left(u_{s, j} E_{k}+u_{s, k} E_{j}\right)+q_{s} \frac{\rho_{s}}{m_{s}}\left(\epsilon_{j \alpha \gamma} u_{s, \alpha} u_{s, k}+\epsilon_{k \alpha \gamma} u_{s, \alpha} u_{s, j}\right) B_{\gamma}+ \\
+\frac{q_{s}}{m_{s}}\left(\epsilon_{j \alpha \gamma} P_{s, \alpha k}+\epsilon_{k \alpha \gamma} P_{s, \alpha j}\right) B_{\gamma}
\end{array}\right)
\end{gathered}
$$

and where $s \in\{$ ion, $e\}$. Maxwell's equations are represented as

$$
\mathbf{U}_{M}=\left(\begin{array}{c}
B_{j} \\
E_{j}
\end{array}\right), \quad \mathbf{F}_{M}=\left(\begin{array}{c}
\epsilon_{j i \alpha} E_{\alpha} \\
-c^{2} \epsilon_{j i \alpha} B_{\alpha}
\end{array}\right), \quad \mathbf{S}_{M}=\left(\begin{array}{c}
0 \\
\frac{e}{\epsilon_{0}}\left(\frac{\rho_{e}}{m_{e}} u_{e, j}-\frac{\rho_{i o n}}{m_{i o n}} u_{i o n, j}\right)
\end{array}\right) .
$$

The above model incorporates extended fluid dynamics equations for each species, along with Maxwell's equations. It should be noted that when taking the velocity moments to arrive at the species transport equations, the third order velocity moment of $\mathcal{G}_{s}$ is zero. This corresponds to having no species heat flux. The individual species solution, flux and source vectors of (5) and (6) are ordered starting with the continuity equation, then the momentum equations, and finally the energy equations, where $\rho_{s}$ is the density, $\mathbf{u}_{s}$ is the bulk velocity, $P_{s, j k}$ is the three dimensional pressure tensor, $q_{s}$ is the charge, $\mathbf{c}_{s}$ is the random velocity vector, and $m_{s}$ is the mass for species $s$. Maxwell's equations given in Eq. (7) include Faraday's and Ampére's laws for the electric field $\mathbf{E}$, and the magnetic field $\mathbf{B}$, respectively, and $\epsilon$ is the permitivity of free space. The source vector of Eq. (6) only includes the non-collision source terms. There are no collision terms related to the Maxwell's equations, and therefore

$$
\mathbf{S}_{M}^{c o l}=0 .
$$

Non-equilibrium collisional processes between the plasma species are represented as Coulomb collisions ${ }^{29}$ and the Bhatnagar, Gross, Krook (BGK) relaxation time approximation ${ }^{30}$ is used to prescribe the collision terms for the ion and electron species as follows:

$$
\mathbf{S}_{s}^{c o l}=\left(\begin{array}{c}
0 \\
\rho_{s} \tilde{\nu}_{s t}\left(u_{t k}-u_{s k}\right) \\
\nu_{s}\left(p_{s} \delta_{j k}-P_{s j k}\right)+2 \frac{\rho_{s} \tilde{\nu}_{s t}}{\left(m_{s}+m_{t}\right)} k_{B}\left(T_{t}-T_{s}\right) \delta_{j k}+ \\
+\frac{m_{t} \rho_{s} \tilde{\nu}_{s t}}{m_{s}+m_{t}}\left[\frac{1}{3} \delta_{j k}\left(\mathbf{u}_{t}-\mathbf{u}_{s}\right)^{2}+\left(u_{t j}-u_{s j}\right)\left(u_{t k}-u_{s k}\right)\right]+ \\
+\rho_{s} \tilde{\nu}_{s t}\left[u_{s k}\left(u_{t j}-u_{s j}\right)+u_{s j}\left(u_{t k}-u_{s k}\right)\right]
\end{array}\right), \quad(s, t) \in\{(e, \text { ion }),(\text { ion }, e)\}
$$


where $\nu_{s}$ is the collision frequency for species $s .{ }^{31}$ The collision frequency, $\nu_{s}$, is the inverse of the collisional relaxation time and is the average number of self collisions for a single particle of species $s$ per second. This governs how quickly a species will approach equilibrium. In addition,

$$
\tilde{\nu}_{s t}=\frac{m_{t}}{m_{s}+m_{t}} \nu_{s t}, \quad T_{s}=\frac{P_{s}}{\rho_{s} \mathrm{R}_{s}},
$$

where $\nu_{s t}$ is the collision frequency for collisions between species $s$ and $t$. The preceding set of coupled partial differential equations describes the transport of fully ionized non-equilibrium anisotropic plasmas, consisting of a single ion species, in the absence of heat flux. It is interesting to note that the coupling of the three sets of equations for the ions, electrons and electromagnetic fields occurs only through the source terms.

\section{Dispersion Analysis of Two-Fluid MHD Model}

In order to better understand the physical and mathematical behavior represented by the system of equations given by Eqs. (3)-(9), and to aid in the development of a numerical solution procedure, a dispersion analysis of the proposed two-fluid model has been carried out. The results of this dispersion analysis are now summarized below.

\section{A. Non-Dimensional Linearized Transport Equations}

The dispersion analysis is applied to the linearized equations. In order to linearize the equations of the two-fluid MHD model, Eq. (3) is first rearranged into a non-conservative form given by

$$
\frac{\partial \mathbf{W}}{\partial t}+\mathcal{A} \frac{\partial \mathbf{W}}{\partial x}+\mathcal{B} \frac{\partial \mathbf{W}}{\partial y}+\mathcal{C} \frac{\partial \mathbf{W}}{\partial z}=\mathcal{S} \mathbf{W}
$$

where the primitive solution vector is

$$
\mathbf{W}=\left(\begin{array}{c}
\mathbf{W}_{i o n} \\
\mathbf{W}_{e} \\
\mathbf{W}_{M}
\end{array}\right), \quad \mathbf{W}_{s}=\left(\begin{array}{c}
\rho_{s} \\
u_{s, x} \\
u_{s, y} \\
u_{s, z} \\
P_{s, x x} \\
P_{s, x y} \\
P_{s, x z} \\
P_{s, y y} \\
P_{s, y z} \\
P_{s, z z}
\end{array}\right), \quad \mathbf{W}_{M}=\left(\begin{array}{c}
B_{x} \\
B_{y} \\
B_{z} \\
E_{x} \\
E_{y} \\
E_{z}
\end{array}\right),
$$

and $s \in\{$ ion, $e\}$. The equations are then non-dimensionalized with respect to the following quantities:

$$
\nu, \quad \rho_{\text {ref }}, \quad p_{\text {ref }}, \quad m_{\text {ref }}, \quad \mu_{0},
$$

which are the interspecies collision frequency, the reference density, the reference isotropic pressure, the reference mass, and the magnetic permitivity of freespace. A reference sound speed can be defined as $a_{r e f}=\sqrt{\gamma \frac{p_{r e f}}{\rho_{r e f}}}$. Using the above quantities, yields the following transformations:

$$
\begin{gathered}
t=\bar{t} \frac{1}{\nu}, \quad x=\bar{x} \frac{a_{r e f}}{\nu} \\
m_{i o n}=\bar{m}_{i o n} m_{r e f}, \quad m_{e}=\bar{m}_{e} m_{r e f}, \quad \rho_{i o n}=\bar{\rho}_{i o n} \rho_{r e f}, \quad \rho_{e}=\bar{\rho}_{e} \rho_{r e f} \\
\mathbf{u}_{i o n}=\overline{\mathbf{u}}_{i o n} a_{r e f}, \quad \mathbf{u}_{e}=\overline{\mathbf{u}}_{e} a_{r e f}, \quad p_{i o n}=\bar{p}_{i o n} \gamma p_{r e f}, \quad p_{e}=\bar{p}_{e} \gamma p_{r e f} \\
\mathbf{E}=\overline{\mathbf{E}} \gamma p_{r e f} \sqrt{\frac{\mu_{0}}{\rho_{r e f}}}, \quad \mathbf{B}=\overline{\mathbf{B}} \sqrt{\mu_{0} \gamma p_{r e f}}, \quad T=\bar{T} \frac{\gamma p_{r e f}}{\rho_{r e f} \mathrm{R}_{i o n}},
\end{gathered}
$$

where the bar indicates the non-dimensional quantity. Please note that in the remainder of this section, the bars have been dropped for simplicity. 
The resulting non-dimensional, non-conservative equations are then linearized about an equilibrium solution state, $\mathbf{W}_{0}$, defined by

$$
\mathbf{W}_{0}=\left(\begin{array}{c}
\mathbf{W}_{0, \text { ion }} \\
\mathbf{W}_{0, e} \\
\mathbf{W}_{0, M}
\end{array}\right), \quad \mathbf{W}_{0, s}=\left(\begin{array}{c}
\rho_{0, s} \\
0 \\
0 \\
0 \\
p_{0, s} \\
0 \\
0 \\
p_{0, s} \\
0 \\
p_{0, s}
\end{array}\right), \quad \mathbf{W}_{0, M}=\left(\begin{array}{c}
B_{0, x} \\
B_{0, y} \\
B_{0, z} \\
0 \\
0 \\
0
\end{array}\right),
$$

where the average velocity, off diagonal pressure terms, and background electric field are assumed to be zero, which are all valid assumptions for quiescent plasmas under equilibrium conditions. The linearization is then achieved by assuming that the solution vector can be approximated by

$$
\mathbf{W}^{*}=\mathbf{W}_{0}+\tilde{\mathbf{W}}
$$

with

$$
\tilde{\mathbf{W}}=\left(\begin{array}{c}
\tilde{\mathbf{W}}_{i o n} \\
\tilde{\mathbf{W}}_{e} \\
\tilde{\mathbf{W}}_{M}
\end{array}\right), \quad \tilde{\mathbf{W}}_{s}=\left(\begin{array}{c}
\tilde{\rho}_{s} \\
\tilde{u}_{s, k} \\
\tilde{P}_{s, j k}
\end{array}\right), \quad \tilde{\mathbf{W}}_{M}=\left(\begin{array}{c}
\tilde{B}_{k} \\
\tilde{E}_{k}
\end{array}\right),
$$

and where $\tilde{\mathbf{W}}$ is the perturbation of the solution from the equilibrium state. The resulting linearized nonconservative equations for $\tilde{\mathbf{W}}$ are as follows:

Non-Dimensional Linearized Multispecies Non-Conservative Continuity

$$
\frac{\partial \tilde{\rho}_{s}}{\partial t}+\rho_{0, s} \frac{\partial \tilde{u}_{s i}}{\partial x_{i}}=0 \quad s \in\{e, i o n\}
$$

Non-Dimensional Linearized Multispecies Non-Conservative Momentum

$$
\begin{gathered}
\frac{\partial \tilde{u}_{s k}}{\partial t}+\frac{1}{\rho_{0, s}} \frac{\partial \tilde{P}_{s i k}}{\partial x_{i}}=\operatorname{Ke} \frac{q_{s}}{m_{s}}\left(\tilde{E}_{k}+\varepsilon_{k \alpha \gamma} \tilde{u}_{s \alpha} B_{0, \gamma}\right)+\tilde{\nu}_{s t}\left(\tilde{u}_{t k}-\tilde{u}_{s k}\right), \\
(s, t) \in\{(e, i o n),(\text { ion }, e)\}
\end{gathered}
$$

Non-Dimensional Linearized Multispecies Non-Conservative Energy

$$
\begin{gathered}
\frac{\partial \tilde{P}_{s j k}}{\partial t}+\delta_{j k} p_{0, s} \frac{\partial \tilde{u}_{s i}}{\partial x_{i}}+\delta_{i j} p_{0, s} \frac{\partial \tilde{u}_{s k}}{\partial x_{i}}+\delta_{i k} p_{0, s} \frac{\partial \tilde{u}_{s j}}{\partial x_{i}}=\operatorname{Ke} \frac{q_{s}}{m_{s}}\left(\varepsilon_{j \alpha \gamma} \tilde{P}_{s \alpha k}+\varepsilon_{k \alpha \gamma} \tilde{P}_{s \alpha j}\right) B_{0, \gamma}+ \\
\quad+\nu_{s}\left(\frac{1}{3}\left(\tilde{P}_{s, x x}+\tilde{P}_{s, y y}+\tilde{P}_{s, z z}\right) \delta_{j k}-\tilde{P}_{s j k}\right)+2\left(\frac{1}{\gamma m_{r e f} \mathrm{R}_{r e f}}\right)+ \\
+2\left(\frac{1}{m_{r e f} \mathrm{R}_{r e f}}\right) \frac{\rho_{0, s} \tilde{\nu}_{s t}}{\left(m_{s}+m_{t}\right)} k_{B}\left[-\frac{p_{0, t}}{\rho_{0, t}^{2} R_{t}} \tilde{\rho}_{t}+\frac{1}{3 \rho_{0, t} R_{t}}\left(\tilde{P}_{t, x x}+\tilde{P}_{t, y y}+\tilde{P}_{t, z z}\right)+\right. \\
\left.+\frac{p_{0, s}}{\rho_{0, s}^{2} R_{s}} \tilde{\rho}_{s}-\frac{1}{3 \rho_{0, s} R_{s}}\left(\tilde{P}_{s, x x}+\tilde{P}_{s, y y}+\tilde{P}_{s, z z}\right)\right] \delta_{j k}, \quad(s, t) \in\{(e, \text { ion }),(\text { ion }, e)\}
\end{gathered}
$$

where the non-dimensional quantity Ke is defined by

$$
\mathrm{Ke}=\frac{e}{\nu m_{\text {ref }}} \sqrt{\gamma \mu_{0} p_{\text {ref }}}
$$

and Maxwell's equations become 
Faraday's Law

$$
\frac{\partial \tilde{\mathbf{B}}}{\partial t}+\left(\frac{\partial \tilde{E}_{z}}{\partial y}-\frac{\partial \tilde{E}_{y}}{\partial z}\right)_{x}-\left(\frac{\partial \tilde{E}_{z}}{\partial x}-\frac{\partial \tilde{E}_{x}}{\partial z}\right)_{y}+\left(\frac{\partial \tilde{E}_{y}}{\partial x}-\frac{\partial \tilde{E}_{x}}{\partial y}\right)_{z}=0
$$

Ampére's Law

$$
\begin{gathered}
\frac{\partial \tilde{\mathbf{E}}}{\partial t}-\frac{c^{2}}{a_{r e f}^{2}}\left(\left(\frac{\partial \tilde{B}_{z}}{\partial y}-\frac{\partial \tilde{B}_{y}}{\partial z}\right)_{x}-\left(\frac{\partial \tilde{B}_{z}}{\partial x}-\frac{\partial \tilde{B}_{x}}{\partial z}\right)_{y}+\left(\frac{\partial \tilde{B}_{y}}{\partial x}-\frac{\partial \tilde{B}_{x}}{\partial y}\right)_{z}\right)= \\
=-\frac{c^{2}}{a_{r e f}^{2}} \operatorname{Ke} n_{i o n} \tilde{\mathbf{u}}_{i o n}+\frac{c^{2}}{a_{r e f}^{2}} \operatorname{Ke} n_{e} \tilde{\mathbf{u}}_{e} .
\end{gathered}
$$

For the dispersion analysis, the following physical values were used to determine the various constants and reference values:

$$
\begin{array}{rlrl}
e & =1.602189246 \times 10^{-19} \mathrm{C} & \gamma & =\frac{5}{3} \\
c & =299792458 \frac{\mathrm{m}}{\mathrm{s}} & m_{\text {ref }} & =m_{\text {ion }}=1.6736 \times 10^{-27} \mathrm{~kg} \\
\mu_{0} & =0.000001256637 \frac{\mathrm{m} \mathrm{kg}}{\mathrm{s}^{2} \mathrm{~A}^{2}} & n_{\text {ref }} & =n_{0}=7.31955 \times 10^{23} \frac{1}{\mathrm{~m}} \\
p_{\text {ref }} & =p_{0}=101325 \frac{\mathrm{N}}{\mathrm{m}^{2}}
\end{array}
$$

and the reference collision frequency was calculated from the ion-electron collision frequency based on the Coulomb collision model as follows: ${ }^{29,32}$

$$
\nu_{s, t}=\frac{4 \sqrt{2 \pi} e_{o}^{4}}{3 k^{\frac{3}{2}}} n_{i o n} Z_{s}^{2} Z_{t}^{2} \ln \Lambda\left(\frac{m_{s}+m_{t}}{m_{s} m_{t}}\right)^{\frac{1}{2}}\left(\frac{m_{s}+m_{t}}{m_{s} T_{t}+m_{t} T_{s}}\right)^{\frac{3}{2}}
$$

where $e_{\circ}$ is the elementary charge constant and $Z_{s}, Z_{t}$ is the charge number for the species $s$ and $t$, respectively, and where the Coulomb logarithm is

$$
\ln \Lambda=23+\frac{3}{2} \ln \left(\frac{T_{e}}{10^{6}}\right)-\frac{1}{2} \ln \left(\frac{n_{e}}{10^{12}}\right) .
$$

\section{B. Eigenvalue Analysis}

Considering initial value problems with planar wave propagation in the $x$-direction only, the perturbative solution vector, $\tilde{\mathbf{W}}$, in Eqs. (18)-(23) can be assumed to have the form

$$
\tilde{\mathbf{W}}=\hat{\mathbf{W}} \exp \left[i\left(\omega \bar{t}^{*}-k \bar{x}^{*}\right)\right]
$$

where $\omega$ is the temporal frequency, and $k$ is the spatial wavenumber. For the initial value problem, $\omega$ is generally complex and $k$ is strictly real valued. This results in the following eigenvalue problem:

$$
\left(i \omega \mathbf{I}-i k \mathcal{A}^{*}-\mathcal{S}^{*}\right) \tilde{\mathbf{W}}=\mathbf{0} .
$$

A number of simplifications are now made which include assuming equal pressures $p_{0, i o n}=p_{0, e}$, that the plasma is a fully ionized quasi neutral plasma $n_{0}=n_{0, i o n}=n_{0, e}$ or $\rho_{0, e}=\frac{m_{e}}{m_{\text {ion }}} \rho_{0, i o n}$, and that the background magnetic field is aligned with the $x$-direction, $\mathbf{B}_{0}=\left(B_{0}, 0,0\right)$. The standard eigenvalue problem can then be formed:

$$
\mathbf{H} \hat{\mathbf{W}}=\omega \hat{\mathbf{W}}
$$

where

$$
\mathbf{H}=k \mathcal{A}^{*}-i \mathcal{S}^{*},
$$

and $k \mathcal{A}^{*}$ and $\mathcal{S}^{*}$ are the linearized, non-dimensional $x$-direction coefficient and source term matrices respectively. 


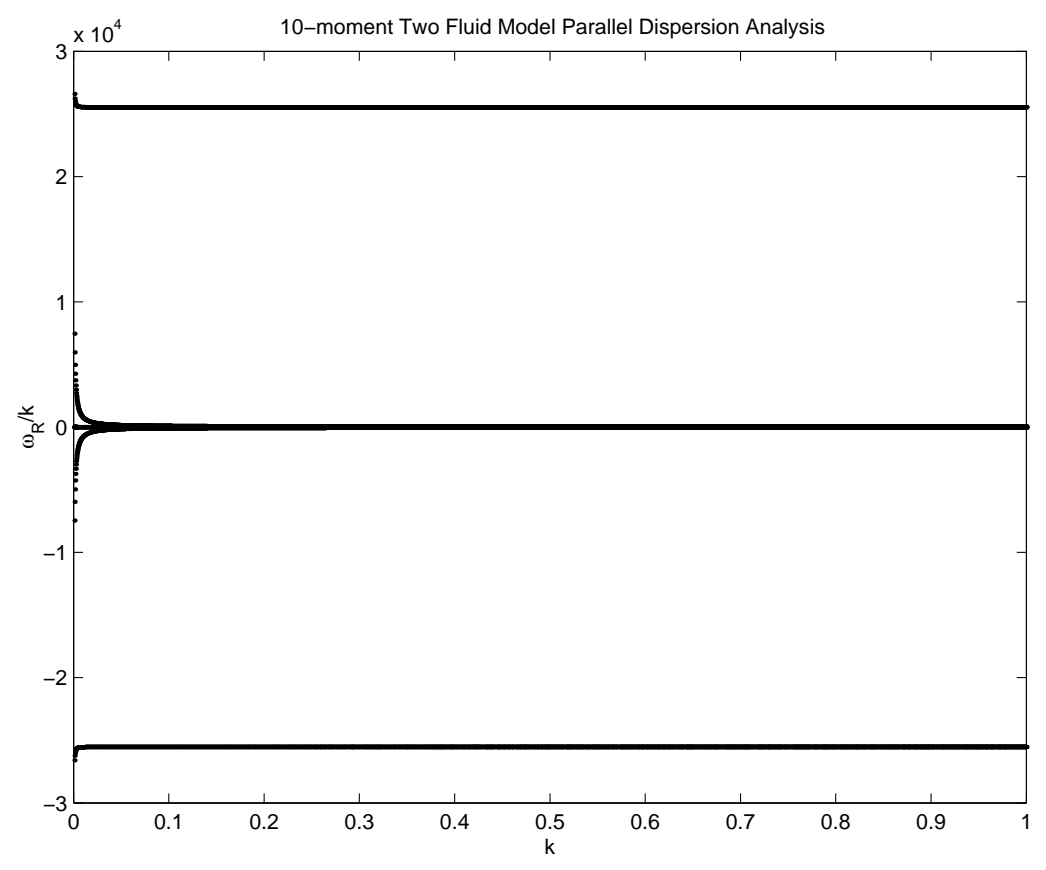

Figure 1. Phasespeed as a function of non-dimensional wavenumber for the parallel direction, indicating behavior of the L- and R-mode waves.

\section{Results of the Dispersion Analysis}

\section{A. Wavespeeds of Fundamental Solution Modes}

The eigenvalue problem of Eq. (29) is solved numerically for $\omega$ for a range of values of $k$. The wave phasespeeds are given by the real part of $\omega$ divided by the spatial wavenumber $k$, and the figures in this section provide the phasespeed, $\frac{\omega_{R}}{k}$, as a function of $k$. In what follows, the various characteristics of the two-fluid MHD model are shown in the Figures 2-11, where the fastest waves will be depicted first, and more of the eigenstructure will be revealed as we telescope or zoom in to investigate the slower wave modes. In addition, the computed wave structure is compared to the dispersive wave behavior calculated for an isotropic 5-moment version of the two-fluid MHD model. The latter is equivalent to the two-fluid model of Shumlak and Loverich with the addition of modelling for interspecies collisions. The comparison with the 5 -moment version of the two-fluid MHD equations is instructive as the high frequency wavespeeds of the 5 -moment model correspond to accepted wavespeeds for the various modes present in ideal MHD. ${ }^{15,22}$

Figure 1 depicts the dispersive wave nature of the two-fluid model showing the full range of wave modes including those with the fastest wavespeeds. Unfortunately, the only waves that can be really seen for this range of velocities and wavenumber are the L- and R-waves, which are the left and right circularly polarized plasma waves respectively. ${ }^{15}$ The fast L- and R-mode waves are composed of a total of four waves at order $10^{4}$ for non-equilibrium conditions or for large values of $k$, which is equivalent to the speed of light following the non-dimensionalization. For small $k$, equilibrium conditions, these waves approach infinite wavespeeds. These waves also agree with the phasespeeds of the L- and R-modes of the 5-moment analysis. The infinite wavespeeds may appear to pose some problems; however, the infinite wavespeeds encountered in this analysis all originate from the electron plasma frequency, which is associated with the plasma cut off frequency. For changes in charge density less than the plasma cut off frequency, the charges will realign to cause Debye shielding, thus masking the electric field due to charge separation. The Debye shielding will stop the propagation of these infinite wavespeeds at equilibrium conditions. Moreover, it should also be noted that it is permissible for an unterminated wave phasespeed to exceed $c$, and that despite an infinite phasespeed, the group velocity of these waves are in fact zero. ${ }^{15}$

In Figures 2 and 3 we are looking at the next lower magnitude waves. These waves are the electron plasma waves which are the same magnitude as those in the 5-moment dispersion analysis. However, the dispersive wave behavior of the 10-moment wave is not the same as that of the 5-moment formulation as 


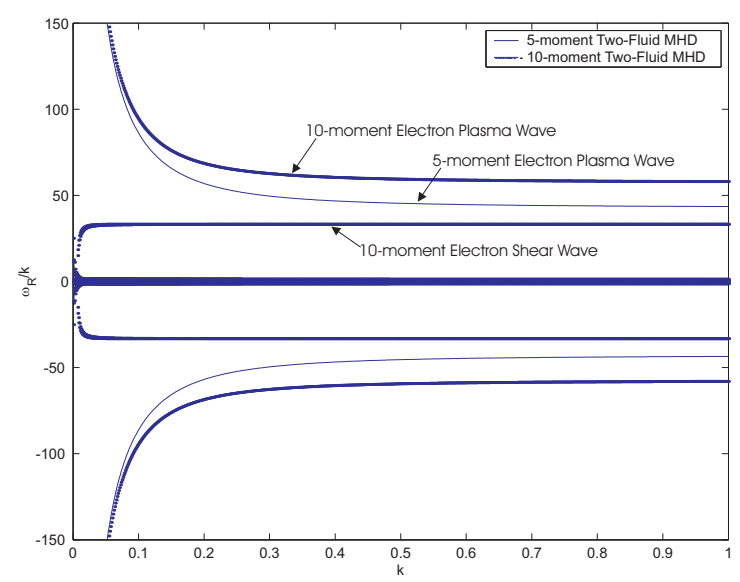

Figure 2. Phasespeed as a function of nondimensional wavenumber for the parallel direction showing electron plasma waves.

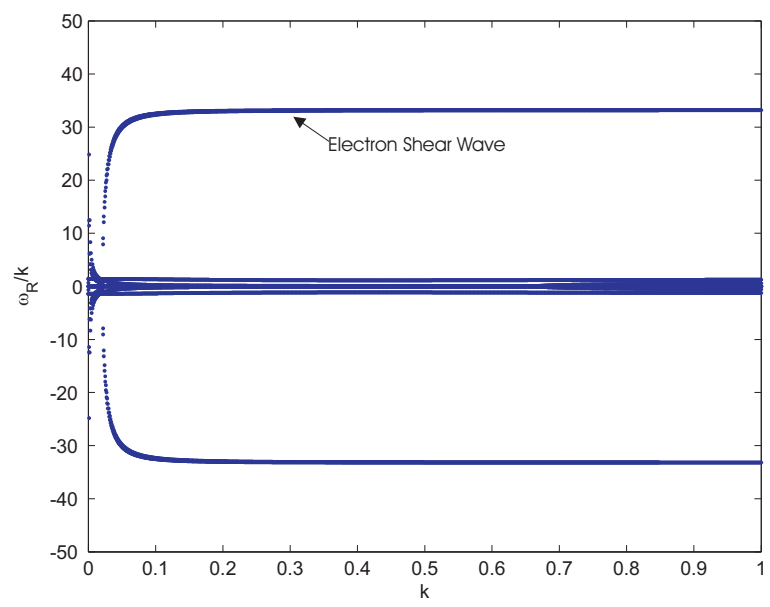

Figure 3. Phasespeed as a function of nondimensional wavenumber for the parallel direction showing electron shear waves.

can be seen in Figure 2. The electron plasma waves are of order 10 for large values of $k$ which is about a thousand times slower than the L- and R-mode waves.

The next wave at about the same magnitude that can be seen in Figure 2 is the electron shear wave. A closer view of the high $k$ behavior of this wave can be seen in Figure 3. In Figure 4, at very small values of $k$ we can see that there is a pair of waves that, at this resolution, appear to be abruptly cut off. This is in fact the equilibrium behavior of the electron shear waves, which can be seen in greater detail in Figures 5 and 7. The electron shear waves have two positive and two negatives waves that have the same phasespeeds at high $k$ as in Figure 3. As $k$ decreases for conditions nearing equilibrium, the two sets of waves (one positive pair and one negative pair) approach the $\omega_{R} / k=0$ axis, as seen in Figure 5. The waves then diverge from each other where one positive and one negative wave mode changes direction and cuts across the zero axis, while the other waves do not change direction, as seen in Figure 7. The resulting behavior of the electron shear waves can be seen in Figure 5 for equilibrium conditions when $k$ is small.

For very small values of $k$, some interesting behavior can be seen from the eight shear waves present, which include not only the electron waves, but also the ion waves which are discussed later in this section. As can be seen in Figure 6, for very small values of $k$, some of the shear wave modes approach a phasespeed of unity. There is some fairly complex structure that can be seen in the behavior of the shear waves. Looking to the non-equilibrium region at high $k$, the waves that do not reverse direction when $k$ decreases, diverge to infinity for very small values of $k$. This behavior is the same for both the electron and ion plasma shear waves. The other wave, the one that cuts across the $\omega_{R} / k=0$ axis then does an arc that returns towards the $\omega_{R} / k=0$ axis as $k$ becomes small, as seen in Figure 5, approaches plus or minus unity for equilibrium conditions. Once again, this is true for both electron and ion plasma waves, even though they greatly differ in scale in the non-equilibrium regime. One positive and one negative wave from the electron and ion shear waves approaches \pm 1 for very small values of $k$ as illustrated in Figure 6 . It shows a strong coupling between the ions and electrons since unity here corresponds to the ion acoustic speed only.

In Figures 8 and 9, we have the Alfvén waves which are one set of waves that do not become infinite for very small values of $k$. Figure 9 shows only the positive Alfvén wave compared to the 5-moment Alfvén wave. We can see that they depart at larger values of $k$. Remember that a value of unity here means ion acoustic velocities, however, we approach ion acoustic velocities due to the non-dimensionalization which defined the magnetic field such that the Alfvén velocity $v_{a l}=\frac{c B_{0} \sqrt{\epsilon_{0}}}{\sqrt{\rho_{r e f}}}$ is equal to the ion acoustic velocity. In the 10-moment formulation, perturbations actually propagate faster than the Alfvén velocity unlike in the isotropic formulation. This is perhaps due to the fact that the Alfvén phasespeed is coupled to perpendicular plasma oscillations and the plasma is freer to oscillate in the 10-moment description which allows for temperature anisotropies, as opposed to the ideal MHD limit where pressures and temperatures are coupled in all directions. This is especially true for non-equilibrium conditions, which is why the Alfvén wavespeed 


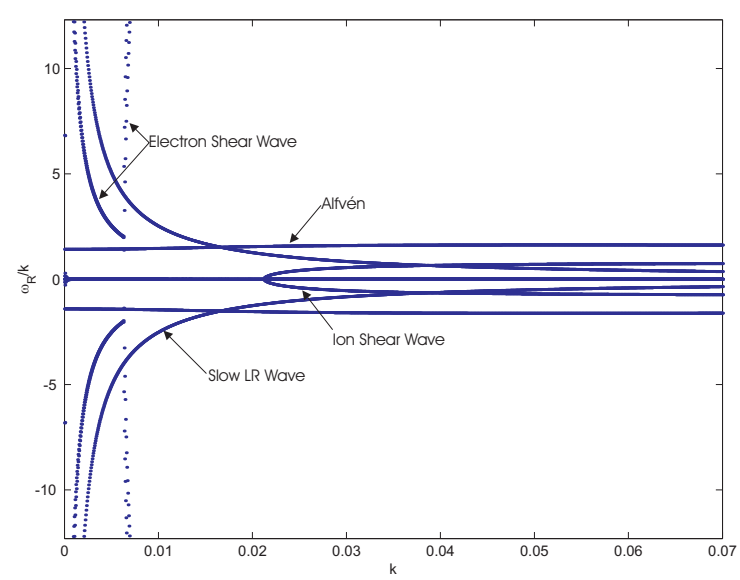

Figure 4. Phasespeed as a function of nondimensional wavenumber showing the dispersive wave behavior of the electron shear waves for small $k$.

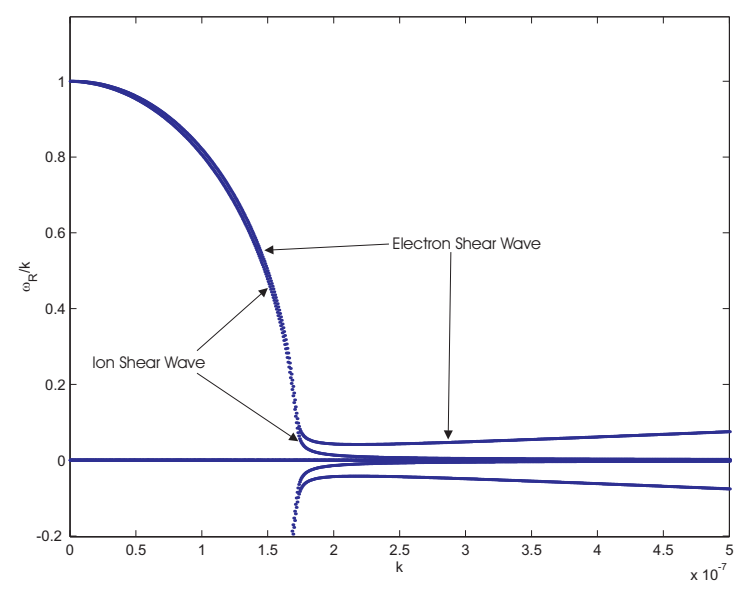

Figure 6. Phasespeed as a function of nondimensional wavenumber showing very small $k$ behavior for electron and ion shear waves for the parallel direction.

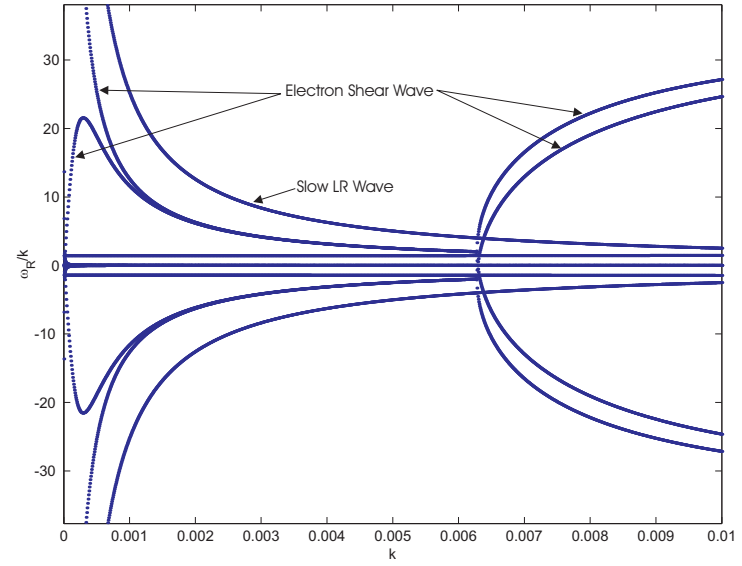

Figure 5. Phasespeed as a function of nondimensional wavenumber showing the dispersive wave behavior of the electron shear waves for very small $k$

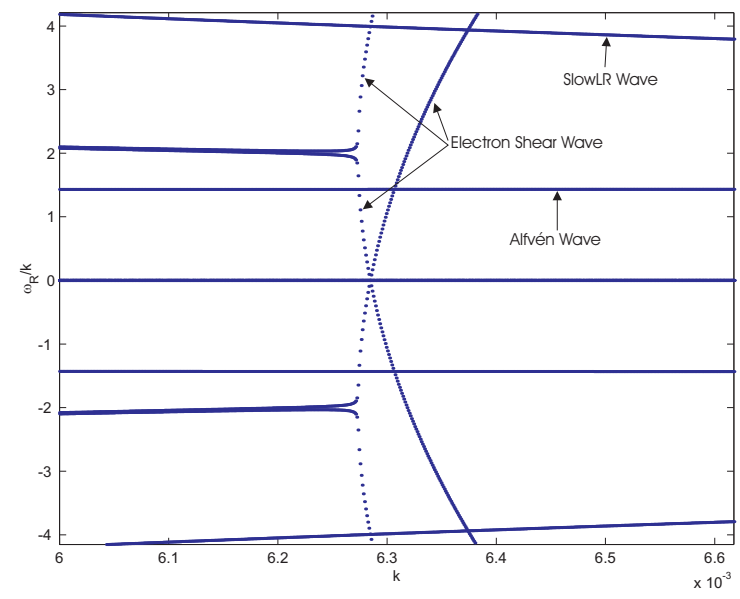

Figure 7. Phasespeed as a function of nondimensional wavenumber showing the dispersive wave behavior of the electron shear waves near the $\omega_{R} / k=0$ axis for small $k$.

diverges rapidly from the 5-moment isotropic result as $k$ increases from zero, and then remains faster, and continues to diverge from the isotropic, near-equilibrium values.

There are two other major waves of interest that can be seen in Figure 8. One is the ion shear waves which have been observed in other previous figures. In Figure 10, the behavior of the four ion shear waves can be seen bracketed within the lower hybrid wave in the small $k$ regime. As was seen in the case of the electron shear waves, the ion shear waves exhibit complex behavior at about $k=0.02$. This is because the phasespeeds shown in Figure 8 decrease and approach the $\omega_{R} / k=0$ axis, where one pair of waves reverse direction and cross the zero axis as the electron shear waves did between $k=0.006$ and $k=0.007$. It is interesting to note that the electron shear waves are much faster than the ion shear waves and asymptote to a constant value at smaller $k$ than the ion shear waves. This means that electrons are much more sensitive to perturbations than ions, and propagate for a wider range of $k$ than the ion shear wave. This makes sense due to their relative mass differences. As in the case of the electron shear waves, the ion shear wave that reverses direction will go through a small arc, that passes very close to the $\omega_{R} / k=0$ axis before approaching a phasespeed of unity for very small values of $k$. The ion shear wave that does not reverse direction coming from non-equilibrium conditions to equilibrium conditions will approach infinity for very small values of $k$. The ion shear waves are generally much slower than the electron shear waves for the entire domain being of 


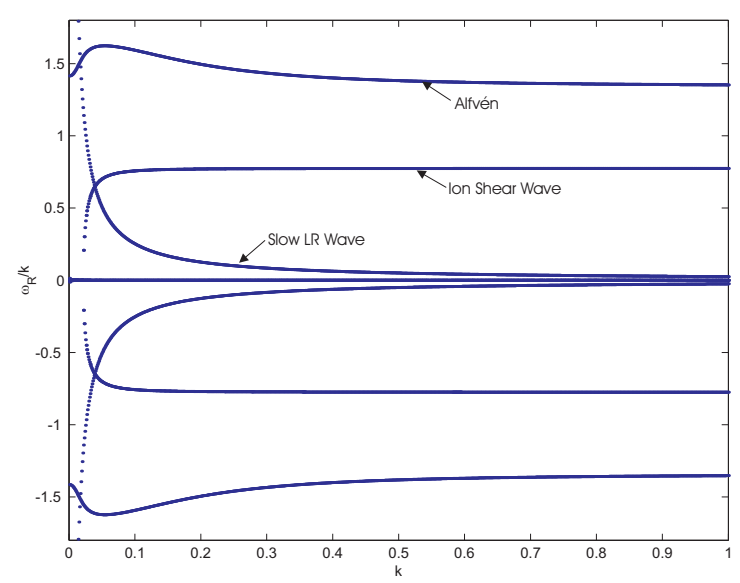

Figure 8. Phasespeed as a function of nondimensional wavenumber showing Alfvén and ion shear waves for the parallel direction

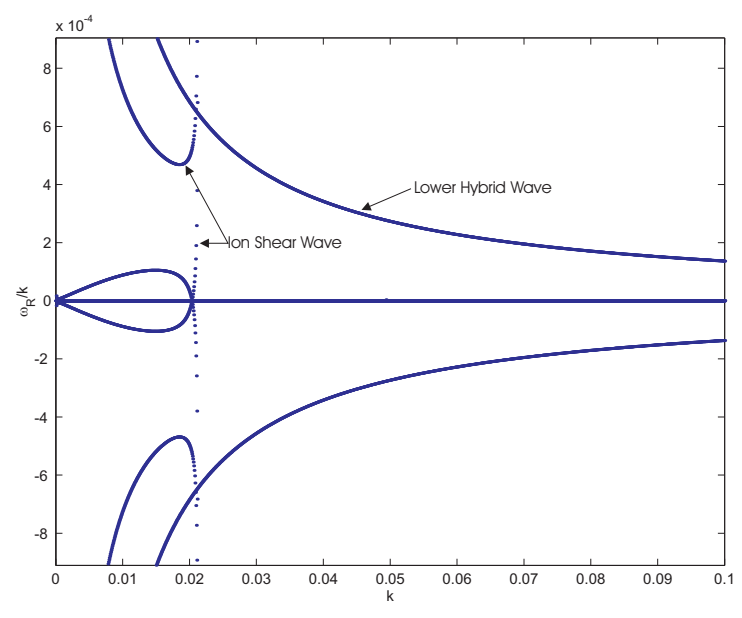

Figure 10. Phasespeed as a function of nondimensional wavenumber showing the lower hybrid and ion shear waves for the parallel direction.

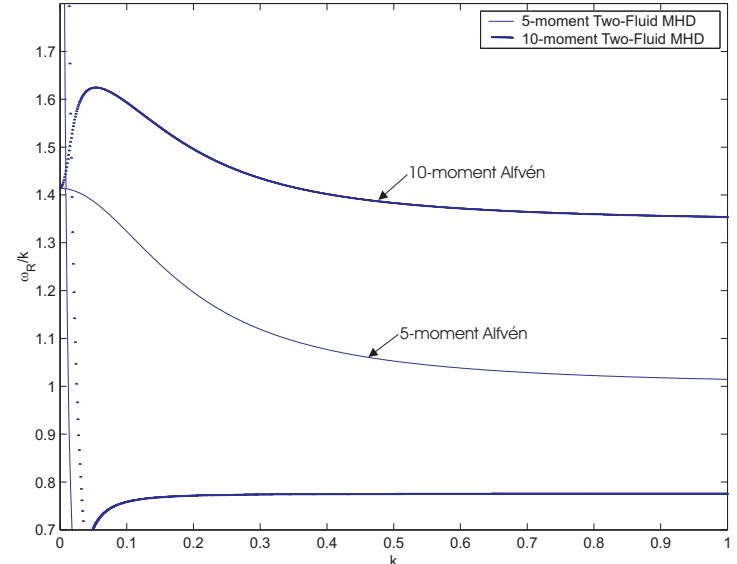

Figure 9. Phasespeed as a function of nondimensional wavenumber showing Alfvén waves for the parallel direction compared to the 5moment dispersion analysis

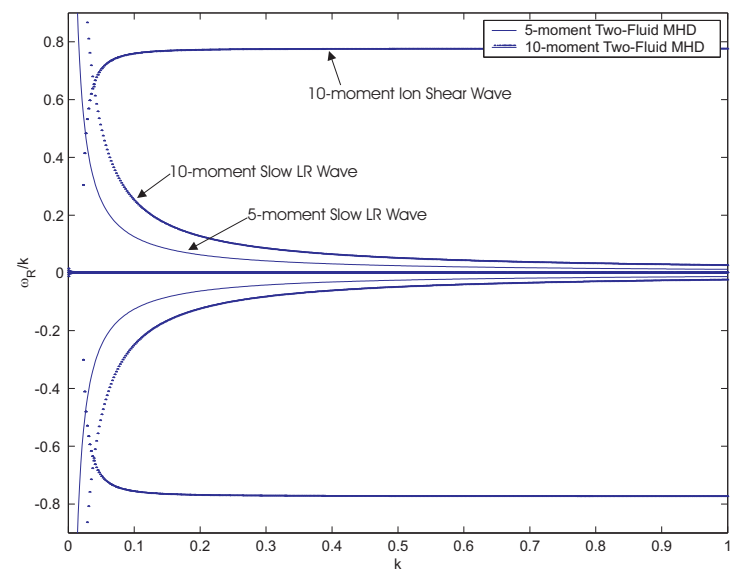

Figure 11. Phasespeed as a function of nondimensional wavenumber showing the slow LR waves for the parallel direction.

order $10^{-1}$ (non-dimensional) for non-equilibrium, two orders of magnitude slower than the electron shear waves which can be attributed to the fact that characteristic speeds tied to movement of particles are usually proportional to $\frac{1}{\sqrt{m}}$ and the ions are four orders of magnitude heavier than electrons.

The next waves encountered are the slow L- and R-mode waves (LR waves), which can both be seen in Figure 11. These waves can be seen with the ion shear waves and are also compared to the 5-moment isotropic result from the 5-moment MHD version of the MHD model. The two sets of slow LR waves do not agree exactly, however, this can be partially attributed to the fact that the LR waves, for the 10-moment formulation, now have a large imaginary component to them. The damping behavior of these waves will be discussed in the next section. The temporal frequency of the slow LR waves are constant and not a function of $k$ and hence the wavespeed will approach zero for very large values of $k$.

The last non-zero wave we will discuss is the lower hybrid wave. This wave can be seen in Figure 10. It is actually the wave that brackets the small $k$ behavior of the ion shear waves. The waves are relatively slow and it should be noted that the temporal frequency is constant and not a function of the spatial wavenumber, the phasespeed goes to zero for very large values of $k$.

There are several zero magnitude waves (eight to be exact). These are waves that simply advect with the flow. These waves include electron and ion entropy, electron and ion transverse pressure waves, and 


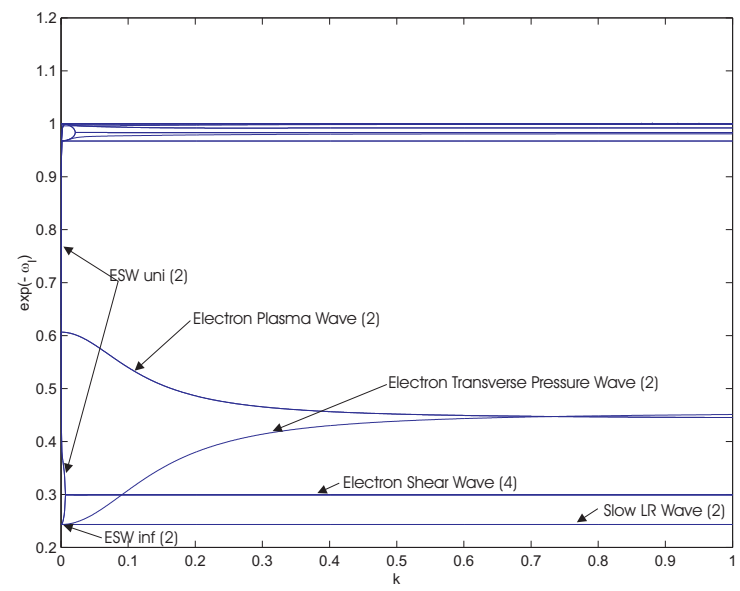

Figure 12. Damping factor as a function of nondimensional wavenumber for each wave mode for the parallel direction.

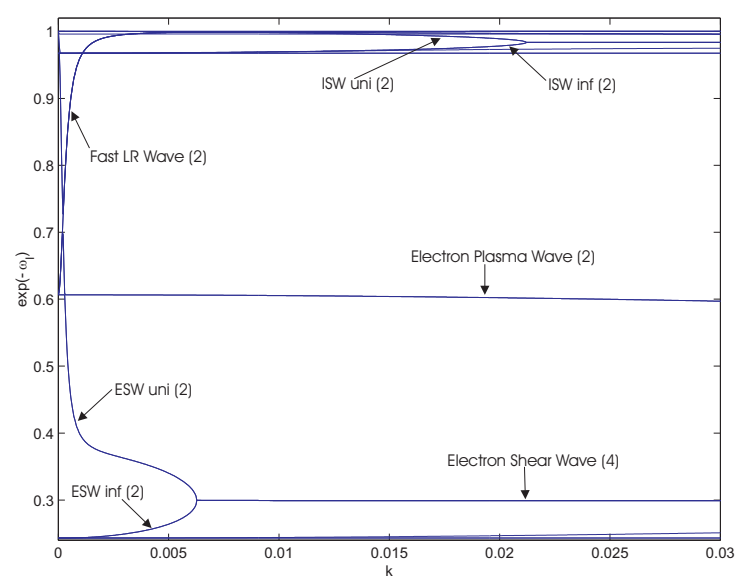

Figure 14. Damping factor as a function of nondimensional wavenumber for small values of $k$ for the parallel direction

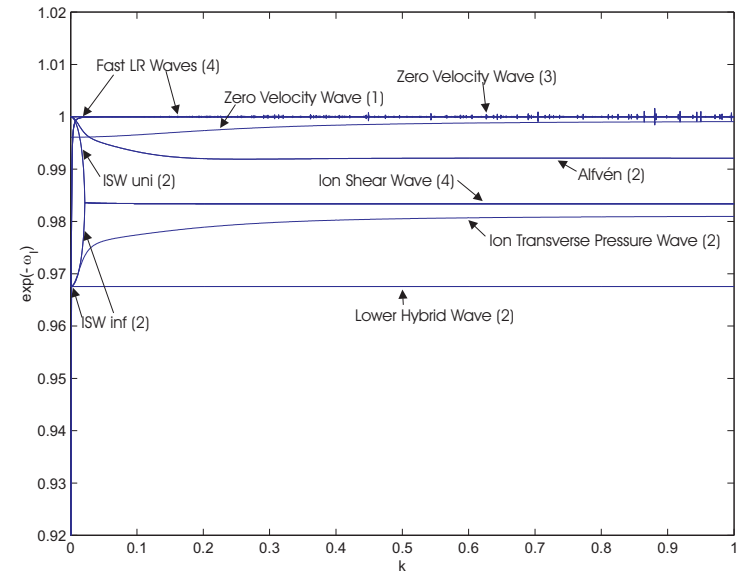

Figure 13. Damping factor as a function of nondimensional wavenumber for the parallel direction showing those modes that exhibit reduced damping.

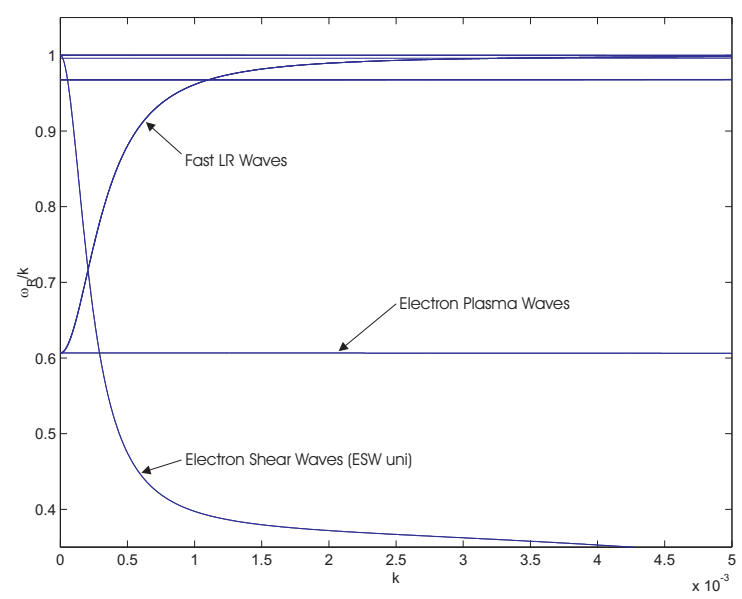

Figure 15. Damping factor as a function of nondimensional wavenumber for very small values of $k$ for the parallel direction.

magnetic and electric flux waves.

As can be seen in the dispersion analysis, the range of speeds over which the various wave modes propagate is very wide. There are large differences in phasespeeds between the EM waves, such as the L- and R-mode waves, and the fluid dynamics waves, as well as between the propagation speeds of the ion and electron waves. These disparate propagation speeds will present some numerical stiffness issues which need to be resolved or mitigated in any solution scheme developed for the solution of the two-fluid MHD model equations. Possible techniques for dealing with the stiffness would include preconditioning methods and the use of implicit time marching schemes.

\section{B. Damping of Fundamental Solution Modes}

In order to explore the damping behavior of the system, the imaginary part of the eigenvalue, $\omega$, has also been analyzed. The damping behavior is dictated by the imaginary part of Eq. (27). Figures 12 and 13 represents the variation of the wave damping as a function of the wavenumber for wavenumbers in the range of 0 to 1 . Figure 13 provides a closer look at the wave modes with lower damping rates. The number in the brackets indicate the number of waves represented by each line.

The high-speed LR waves appear to have no damping for this range. In actual fact, for very small 
values of $k$, or equilibrium conditions, when LR has infinite wavespeeds, there is heavy damping which is approximately 0.6 or an $\omega_{I}$ of 0.5 as can be seen in Figure 15. This behavior helps explain why we do not observe infinite waves in physical plasmas.

The Alfvén wave is less damped at near equilibrium conditions. At very small values of $k$ it is not damped at all. Similarly, the electron plasma wave is also less damped nearer to equilibrium, but still remains damped for small values of $k$. This means perturbations and non-equilibrium disturbances are more damped than equilibrium waves as can be expected of magneto-acoustic phenomenon.

There is an interesting phenomenon in both the ion and electron shear waves. For non-equilibrium values at large $k$, both sets of waves have a constant damping, where the electron shear waves have a much higher damping than the ions. This is likely due to the fact that electrons are much faster and lighter than the ions and thus, travel faster and have higher damping when encountering other particles. For near equilibrium conditions (i.e., small $k$ ), both sets of the four shear waves split off into two sets of two waves which can be seen in Figure 14. The split off point for the electron shear waves coincides with the phenomenon observed in Figure 4. The ion shear waves exhibit a similar divergence which occurs at higher wavenumbers. This is the same point at which one set diverges to infinite phasespeeds while the other set of waves cross the zero axis and eventually approaches a phasespeed of unity. The labels ESW uni and ISW uni indicate the two waves that approach unity and ESW inf and ISW inf indicate the waves that approach infinity. The modes that approach unity actually end up as undamped waves for very small values of $k$. The ESW uni waves become undamped in Figure 15. This indicates that these ion acoustic speed waves (ion acoustic even though there are both electrons and ions travelling at this speed) that propagate pressure shears are undamped at equilibrium. As mentioned before, at equilibrium conditions, there is a strong coupling between the ions and electrons, through the electric fields, causing them to propagate together at a single velocity, which is the ion acoustic velocity. The ion behavior predominates because the mass of the ions cause their properties to dominate over the much lighter electrons. Conversely, at non-equilibrium conditions, there is no coupling between the ions and electrons because there is insufficient time to compensate for local perturbations. For the waves that approach infinite wavespeeds, we see that they are heavily damped. Once again, this helps mitigate the very fast wave speeds encountered. For the waves that approach infinity for small values of $k$, the electron waves are much more damped compared to the ions. Again, this is probably due to their relative masses. It should be noted that the shear waves for the ions and electrons have a similar structure to the shear waves of the 10 -moment analysis ${ }^{33}$ for non-magnetized non-equilibrium gases. The differences come from the non-dimensionalization used as well as the presence of two fluids coupled through electromagnetic forces.

The electron plasma waves have less damping at equilibrium than at high $k$. Since the electron plasma wave is associated with thermal movements of electrons transmitting electron cyclotron information, the electron shielding at equilibrium masks strong charges while at high frequency spatial wave numbers there is less shielding resulting in strong charges and thus large damping due to coulomb collisions with other particles including the large ions impeding the motion of thermal electrons. On the other hand, the lower hybrid wave, which is an ion wave related to the ion plasma frequency, exhibits a constant damping rate for the full range of $k$ considered.

The slow LR waves have the highest damping of all waves for the entire range of $k$. Some other waves that are present are the ion and electron transverse pressure waves which are zero velocity waves. Again the electron waves are more highly damped than the ions. There are also three undamped zero waves and one slightly damped wave. They appear to be linear combinations of transverse pressure, entropy, and parallel $\mathrm{E}$ and $\mathrm{B}$ flux waves, as determined from numerical evaluation of the corresponding eigenvectors.

\section{Numerical Solution Scheme}

In order to investigate further properties of the two-fluid model, a numerical solution scheme is proposed and developed for the solution of the one-dimensional form of Eqs. (3)-(9). In particular, a higher-order Godunov-type upwind finite-volume scheme ${ }^{34}$ is used to solve the two-fluid MHD model equations. Schemes of this type are appropriate for hyperbolic systems of equations. In the proposed approach, numerical fluxes are evaluated using the Riemann solver based flux function of Harten, Lax, van Leer, and Einfeldt (HLLE). ${ }^{35}$ Higher-order accuracy (second-order for smooth solutions) is achieved via piecewise linear reconstruction in conjunction with a Barth-Jesperson ${ }^{36}$ slope limiter. A fully implicit time marching scheme is used to integrate the resulting coupled system of ordinary differential equations that results from the finite volume 
spatial discretization procedure. A second-order-backwards difference time marching scheme with a dual time stepping ${ }^{37}$ procedure is used to compute higher-order unsteady solutions.

\section{A. Implicit Time Marching Scheme}

As a first step towards developing a numerical solution scheme for the multi-fluid plasma model, a finitevolume scheme with a first-order implicit time marching scheme will be considered (this scheme is also used for the discrete dispersion analysis of the next section). Using an implicit Euler time marching scheme and a uniform spatial mesh with $x_{i}=x_{0}+i \Delta x$ and $\Delta x=x_{i+1}-\Delta x_{i}=$ constant, the following fully discrete finite-volume scheme can be obtained:

$$
\mathbf{U}_{i}^{n+1}=\mathbf{U}_{i}^{n}-\frac{\Delta t}{\Delta x}\left(\mathcal{F}_{i+\frac{1}{2}}^{n+1}-\mathcal{F}_{i-\frac{1}{2}}^{n+1}\right)+\Delta t \mathbf{S}_{i}^{n+1} .
$$

In the above equation, $\mathbf{U}_{i}^{n}$ is the conservative solution state vector defined in Eqs. (3)-(7) for time level $n$ and cell $i, \mathcal{F}_{i \pm \frac{1}{2}}^{n}$ is the interface flux at time level $n$ and interface $i \pm \frac{1}{2}, \mathbf{S}_{i}^{n}$ is the source term for time level $n$ and cell $i$, and $\Delta t$ is the time step. The flux functions and source terms can be linearized to arrive at the following block tridiagonal system of equations:

$$
\begin{gathered}
\left(\mathbf{I}+\frac{\Delta t}{\Delta x} \frac{\partial \mathcal{F}_{i+\frac{1}{2}}^{n}}{\partial \mathbf{U}_{i}}-\frac{\Delta t}{\Delta x} \frac{\partial \mathcal{F}_{i-\frac{1}{2}}^{n}}{\partial \mathbf{U}_{i}}-\Delta t \frac{\partial \mathbf{S}_{i}^{n}}{\partial \mathbf{U}_{i}}\right) \Delta \mathbf{U}_{i}^{n}+\frac{\Delta t}{\Delta x}\left(\frac{\partial \mathcal{F}_{i+\frac{1}{2}}^{n}}{\partial \mathbf{U}_{i+1}} \Delta \mathbf{U}_{i+1}^{n}-\frac{\partial \mathcal{F}_{i-\frac{1}{2}}^{n}}{\partial \mathbf{U}_{i-1}} \Delta \mathbf{U}_{i-1}^{n}\right)= \\
=-\frac{\Delta t}{\Delta x}\left(\mathcal{F}_{i+\frac{1}{2}}^{n}-\mathcal{F}_{i-\frac{1}{2}}^{n}\right)+\Delta t \mathbf{S}_{i}^{n}
\end{gathered}
$$

where $\Delta \mathbf{U}_{i}^{n}=\mathbf{U}_{i}^{n+1}-\mathbf{U}_{i}^{n}$. The above equation can be re-expressed as

$$
\left(\mathbf{I}-\Delta t \frac{\partial \mathbf{R}\left(\mathbf{U}^{n}\right)}{\partial \mathbf{U}^{n}}\right) \frac{\Delta \mathbf{U}^{n}}{\Delta t}=\mathbf{R}\left(\mathbf{U}^{n}\right),
$$

where $\frac{\partial \mathbf{R}\left(\mathbf{U}^{n}\right)}{\partial \mathbf{U}^{n}}$ is the banded block tridiagonal matrix

$$
\frac{\partial \mathbf{R}\left(\mathbf{U}^{n}\right)}{\partial \mathbf{U}^{n}}=\mathbf{B}\left(\frac{1}{\Delta x} \frac{\partial \mathcal{F}_{i-\frac{1}{2}}^{n}}{\partial \mathbf{U}_{i-1}},\left(\frac{1}{\Delta x} \frac{\partial \mathcal{F}_{i-\frac{1}{2}}^{n}}{\partial \mathbf{U}_{i}}-\frac{1}{\Delta x} \frac{\partial \mathcal{F}_{i+\frac{1}{2}}^{n}}{\partial \mathbf{U}_{i}}+\frac{\partial \mathbf{S}_{i}^{n}}{\partial \mathbf{U}_{i}}\right),-\frac{1}{\Delta x} \frac{\partial \mathcal{F}_{i+\frac{1}{2}}^{n}}{\partial \mathbf{U}_{i+1}}\right),
$$

and $\mathbf{R}\left(\mathbf{U}^{n}\right)$ is the solution residual given by

$$
\mathbf{R}\left(\mathbf{U}^{n}\right)=\left(-\frac{1}{\Delta x}\left(\mathcal{F}_{i+\frac{1}{2}}^{n}-\mathcal{F}_{i-\frac{1}{2}}^{n}\right)+\Delta t \mathbf{S}_{i}^{n}\right) .
$$

The interface fluxes $\mathcal{F}_{i \pm \frac{1}{2}}^{n}$ are computed using an HLLE flux function ${ }^{35}$ where the interface solution states are determined using piecewise linear spatial reconstruction with a Barth-Jesperson slope limiter. ${ }^{36}$ The above fully describes a first-order in time, second-order in space, finite-volume implicit time marching scheme.

\section{B. Implicit Dual Time Stepping with Second-Order Backwards Time Marching}

The complete high-order implicit scheme of the two-fluid MHD model is now described. Higher-order accuracy in both space and time was achieved by adopting a dual time formulation:

$$
\frac{\mathrm{d} \mathbf{U}}{\mathrm{d} t}+\frac{\mathrm{d} \mathbf{U}}{\mathrm{d} \tau}=\mathbf{R}(\mathbf{U}),
$$

where $t$ is the physical time and $\tau$ is a pseudo-time so that we can write

$$
\frac{\mathrm{d} \mathbf{U}}{\mathrm{d} \tau}=\mathbf{R}(\mathbf{U})-\frac{\mathrm{d} \mathbf{U}}{\mathrm{d} t}=\mathbf{R}^{*}(\mathbf{U})
$$

which can be driven to steady state in $\tau$ or

$$
\frac{\mathrm{d} \mathbf{U}}{\mathrm{d} \tau}=\mathbf{R}^{*}(\mathbf{U})=\mathbf{0}
$$


For the proposed scheme, an implicit second-order backwards differencing discretization is used for the derivative with respect to physical time, $t$, and an implicit Euler discretization was used for the pseudo-time, $\tau$. We therefore arrive at:

$$
\frac{\Delta \mathbf{U}^{n+k}}{\Delta \tau}=\mathbf{R}\left(\mathbf{U}^{n+k+1}\right)-\left[\frac{\frac{3}{2} \mathbf{U}^{n+k+1}-2 \mathbf{U}^{n}+\frac{1}{2} \mathbf{U}^{n-1}}{\Delta t}\right],
$$

where $\Delta \mathbf{U}^{n+k}=\mathbf{U}^{n+k+1}-\mathbf{U}^{n+k}$. The physical time residual $\mathbf{R}\left(\mathbf{U}^{n+k+1}\right)$ and the $\mathbf{U}^{n+k+1}$ is linearized to arrive at:

$$
\left[\left(1+\frac{3}{2} \frac{\Delta t}{\Delta \tau}\right) \mathbf{I}-\Delta \tau \frac{\partial \mathbf{R}\left(\mathbf{U}^{n+k}\right)}{\partial \mathbf{U}^{n+k}}\right] \frac{\Delta \mathbf{U}^{n+k}}{\Delta \tau}=\mathbf{R}\left(\mathbf{U}^{n+k}\right)-\left[\frac{\frac{3}{2} \mathbf{U}^{n+k}-2 \mathbf{U}^{n}+\frac{1}{2} \mathbf{U}^{n-1}}{\Delta t}\right],
$$

where $\mathbf{R}\left(\mathbf{U}^{n+k}\right)$ and $\frac{\partial \mathbf{R}\left(\mathbf{U}^{n+k}\right)}{\left.\partial \mathbf{U}^{n+k}\right)}$ are Eqs. (35) and (34) respectively. The HLLE flux function and piecewise spatial reconstruction with Barth-Jesperson slope limiting is once again used to solve the interface fluxes. The physical time step $\Delta t$ in the expressions above is determined through a Time Step Control Factor (TSCF) relation where the TSCF number is a non-dimensional parameter that controls the size of the time step as follows:

$$
\mathrm{TSCF}=\frac{\Delta t}{\min \left(\frac{\Delta x}{c}, \frac{1}{\omega_{p e}}\right)}
$$

where $c$ is the speed of light and $\omega_{p e}$ is the electron plasma frequency $\omega_{p e}=\sqrt{\frac{n_{e} e^{2}}{m_{e} \epsilon_{\circ}}}$. The inclusion of the plasma frequency in the definition of the TSCF parameter is a common limiting time scale in the numerical simulation of plasmas. ${ }^{38}$ For an explicit scheme, there is a condition on the stability of the scheme, namely

$$
\Delta t \leq \min \left(\frac{\Delta x}{c}, \frac{1}{\omega_{p e}}\right)
$$

where a TSCF of unity represents the boundary between unstable (TSCF $>1.0$ ) and stable (TSCF $\leq 1.0)$ explicit schemes. The pseudo-time step $\Delta \tau$ is also determined using a pseudo TSCF or sub iteration TSCF which is defined by the same relation, but for the pseudo time step.

\section{Discrete Dispersion Analysis}

In order to gain a better understanding of the capabilities of the proposed numerical scheme described above, a dispersion analysis of the discretized form of the two-fluid MHD model equations has been performed. A summary of the results of the discrete dispersion analysis now follows.

\section{A. Linearized Solution Scheme}

Following the procedure outlined in the dispersion analysis above, a linearized non-dimensional form of the one-dimensional conservative transport Eqs. (3)-(9) can be written as

$$
\frac{\partial \mathbf{U}^{*}}{\partial t}+\frac{\partial}{\partial x} \mathbf{F}_{x}^{*}=\mathbf{S}^{*}
$$

where, in this case, the equilibrium state used in the linearization process, $\mathbf{U}_{0}$, is the same as that used for the dispersion analysis of the non-discretized form of the equations. The finite volume solution of the linearized equation is considered for a uniform mesh as with the proposed numerical scheme with $x_{i}=x_{0}+i \Delta x$ and $\Delta x=x_{i+1}-\Delta x_{i}=$ constant. Applying an explicit Euler time marching scheme along with a first-order version of the spatial discretization procedure defined above, the linearized non-dimensional form of the two-fluid MHD model equations results in the following fully discrete solution update scheme:

$$
\left(\tilde{\mathbf{W}}_{i}^{n+1}-\tilde{\mathbf{W}}_{i}^{n}\right)=\frac{\Delta t}{2 \Delta x}\left[\mathbf{F}_{c o}\left(\tilde{\mathbf{W}}_{i-1}^{n}-\tilde{\mathbf{W}}_{i+1}^{n}\right)+\mathbf{A}\left(\tilde{\mathbf{W}}_{i-1}^{n}-2 \tilde{\mathbf{W}}_{i}^{n}+\tilde{\mathbf{W}}_{i+1}^{n}\right)\right]+\Delta t \mathbf{S}_{c o} \tilde{\mathbf{W}}_{i}^{n}
$$

where $\tilde{\mathbf{W}}_{i}^{n}$ is simply the perturbative primitive solution state vector Eq. (16) for time level $n$ and cell $i$, $\mathbf{F}^{*}=\mathbf{F}_{c o} \tilde{\mathbf{W}}$ and $\mathbf{S}^{*}=\mathbf{S}_{c o} \tilde{\mathbf{W}}$ which relate the flux and source terms to the perturbative solution vector 
through coefficient matrices, and $\Delta t$ is the time step. The diagonal matrix $\mathbf{A}$ contains the local numerical maximum wavespeeds of the system as a result of the HLLE Riemann flux function. If an implicit Euler time marching scheme is used, the set of fully discrete equations becomes:

$$
\left(\tilde{\mathbf{W}}_{i}^{n+1}-\tilde{\mathbf{W}}_{i}^{n}\right)=\frac{\Delta t}{2 \Delta x}\left[\mathbf{F}_{c o}\left(\tilde{\mathbf{W}}_{i-1}^{n+1}-\tilde{\mathbf{W}}_{i+1}^{n+1}\right)+\mathbf{A}\left(\tilde{\mathbf{W}}_{i-1}^{n+1}-2 \tilde{\mathbf{W}}_{i}^{n+1}+\tilde{\mathbf{W}}_{i+1}^{n+1}\right)\right]+\Delta t \mathbf{S}_{c o} \tilde{\mathbf{W}}_{i}^{n+1},
$$

which is the linearized first order version of our numerical solution scheme described in the previous section.

\section{B. Discrete Eigenvalue Analysis}

For the discrete eigenstructure, the following trial solution is used for $\tilde{\mathbf{W}}$ in Eqs. (44) and (45):

$$
\tilde{\mathbf{W}}_{j}^{n}=Z^{n} \exp (i j k \Delta x) \hat{\mathbf{W}} .
$$

Also,

$$
Z=(\exp [i \omega \Delta t])=e^{-\omega_{I} \Delta t}\left(\cos \omega_{R} \Delta t+i \sin \omega_{R} \Delta t\right)
$$

where $\omega_{R}$ and $\omega_{I}$ are the real and imaginary parts of $\omega$ respectively. Substitution of Eq. (46) into Eqs. (44) and (45) results in a set of linear equations that can be expressed as an eigenvalue problem of the form

$$
\mathbf{H} \hat{\mathbf{W}}=Z \hat{\mathbf{W}}
$$

where

$$
\mathbf{H}=\mathbf{I}+\frac{\Delta t}{\Delta x} \frac{1}{2}\left[\mathbf{F}_{c o}\left(\mathrm{e}^{-i k \Delta x}-\mathrm{e}^{i k \Delta x}\right)+\mathbf{A}\left(\mathrm{e}^{-i k \Delta x}-2+\mathrm{e}^{i k \Delta x}\right)\right]+\Delta t \mathbf{S}_{c o}
$$

and

$$
\mathbf{H}=\left[\mathbf{I}-\frac{\Delta t}{\Delta x} \frac{1}{2}\left[\mathbf{F}_{c o}\left(\mathrm{e}^{-i k \Delta x}-\mathrm{e}^{i k \Delta x}\right)+\mathbf{A}\left(\mathrm{e}^{-i k \Delta x}-2+\mathrm{e}^{i k \Delta x}\right)\right]-\Delta t \mathbf{S}_{c o}\right]^{-1},
$$

for the explicit and implicit Euler time marching schemes respectively.

The time step, $\Delta t$, is determined in the same manner as the physical time step defined by Eq. (41) through the TSCF parameter. The implicit scheme is unconditionally stable, at least for the linearized problem.

\section{Results of Discrete Dispersion Analysis}

The eigenproblem of Eq. (49) is solved numerically for $Z$ for a range of $k$ values. Results for both the explicit and implicit schemes will be presented, and the recovery of the analytic dispersive behavior and the stability of the discrete system of equations are examined.

\section{A. Dispersion of the Finite-Volume Discretization with Explicit Time Marching Scheme}

The dispersion of the discrete system with explicit time marching scheme has been explored by plotting the eigenvalues of the eigenvalue problem for four separate values of $\Delta x$ ranging from $\Delta x=1.0$ to $\Delta x=0.001$. The value of the TSCF parameter in all cases is 0.1. The discretized scheme is valid for $k$ values up to $k_{\max }=\frac{\pi}{\Delta x}$, and a range of $k$ up to 314 is shown to give a better illustration of the phasespeed behavior showing the full range of validity for the $\Delta x=0.01$ case with the other cases for comparison. It should be noted that all of the following figures were stable in the imaginary plane as $\Delta t$ satisfies the stability criteria of Eq. (42). The results of the eigensystem analysis are shown in Figures 16 and 17.

As can be seen in the figures, as the $\Delta x$ value becomes smaller, the wavespeeds approach the expected analytical wavespeeds described and discussed earlier in the paper. Figure 16 shows the fastest L- and Rmode waves. In Figure 17, we have reduced the scale of $k$ to something closer to our previous analysis and the behavior of the Alfvén waves can be seen as well as those of the ion shear wave and the slow L- and $\mathrm{R}$-mode waves. Once again, the phasespeeds approach the analytical values as $\Delta x$ becomes small. 


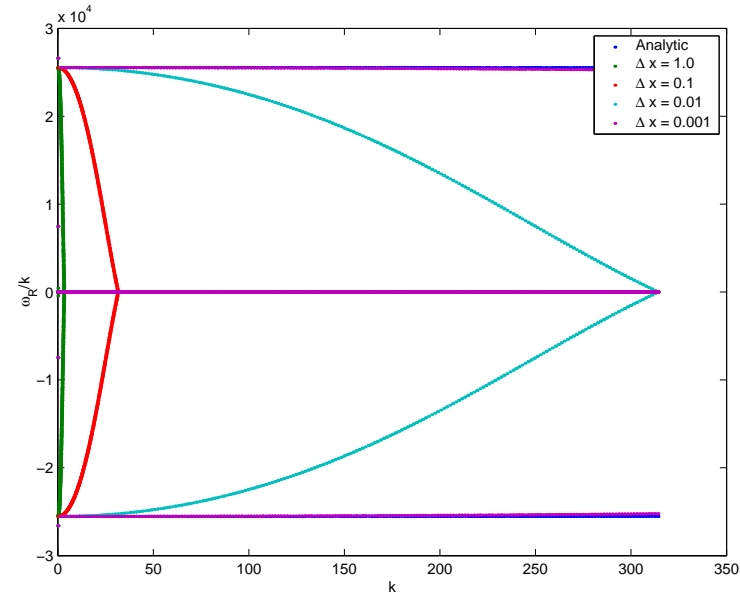

Figure 16. Phasespeed as a function of nondimensional wavenumber for various $\Delta x$ for the parallel direction (L- and R-mode waves visible).

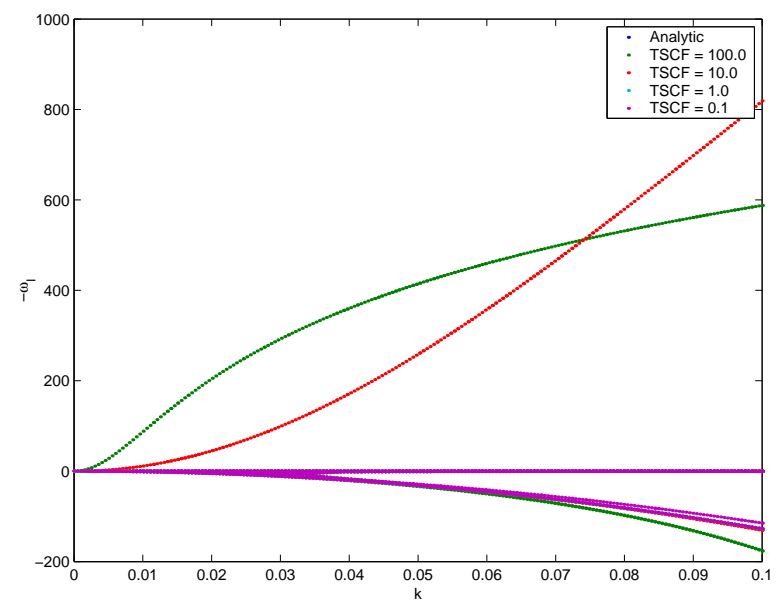

Figure 18. Stability of the explicit scheme for various TSCF for the parallel direction

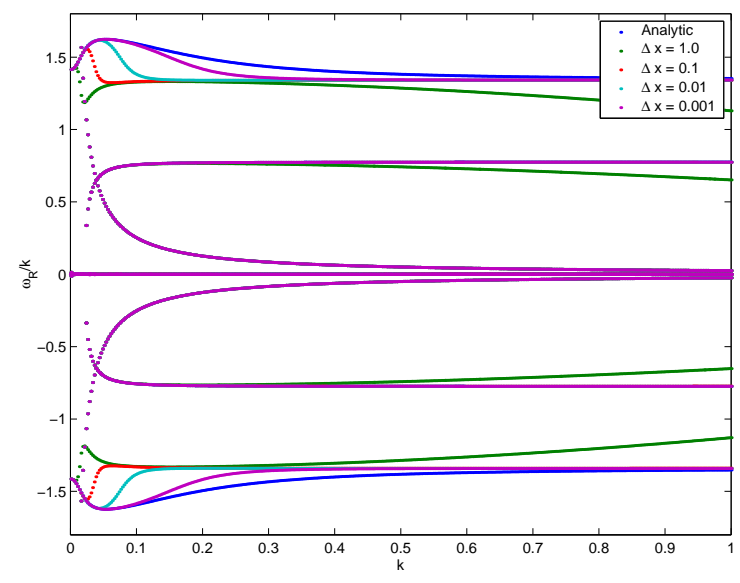

Figure 17. Phasespeed as a function of nondimensional wavenumber for various $\Delta x$ for the parallel direction.

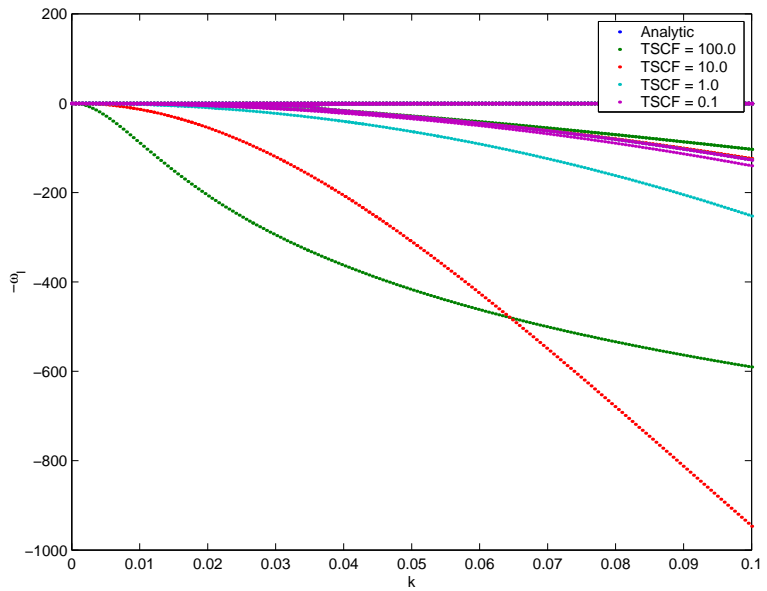

Figure 19. Stability of the implicit scheme for various TSCF for the parallel direction

\section{B. Stability of the Finite-Volume Discretization with Explicit Time Marching Scheme}

Next the stability of the finite-volume discretization with the explicit time marching scheme is examined. The stability of the solution scheme is dictated by the imaginary component of the eigenvalues. To view the unstable regions with greater ease, $-\omega_{I}$ as a function of non-dimensional wavenumber $k$ is considered.

Figure 18 depicts the stability of the proposed solution method for when the condition of Eq. (42) is not satisfied. A value of unity is used for $\Delta x$. and the analysis is performed for various TSCF values ranging from 100.0 to 0.1. As can be seen in Figure 18, the discrete equations become unstable when TSCF is greater than unity, for which the time steps violate the stability condition of Eq. (42). As the TSCF number becomes smaller, bringing the time step into the stable region, the imaginary component of $\omega$ moves to the stable region (i.e., $-\omega_{I} \leq 0$ ). The time step used for a TSCF of 1.0 is $\Delta t=3.9 \times 10^{-5}$. Note that the time is non-dimensionalized according to $t=\bar{t} \frac{1}{\nu}$ where $\nu$ is of order $10^{12} \mathrm{~s}^{-1}$. This would require $\Delta t$ of order $10^{-17} \mathrm{~s}$ to be stable. This is much smaller than other time scales associated with convection, acoustical propagation and collisional processes. This is where the stiffness arises. The electron plasma frequency dictates the numerical time step that can be taken, which in many cases, is much smaller than the time scales of the phenomenon that are often of primary interest.

In summary, it has been shown that an accurate numerical solution procedure for the two-fluid MHD model can be achieved as $\Delta x$ becomes small. However, stability of the explicit time marching scheme is 


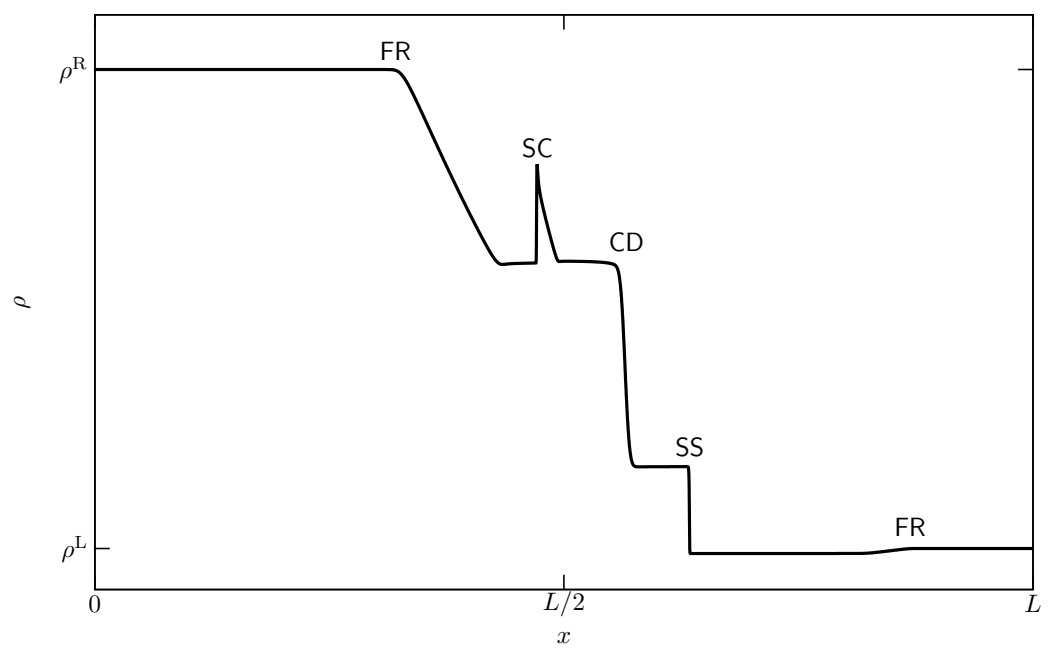

Figure 20. Density profile of the ideal MHD Brio-Wu solution.

subject to the stability condition of Eq. (42). This stability condition can result in a very stiff solution scheme if one is interested in only accurately resolving solution content associated with plasma convection, acoustical, and particle collisional processes, as is commonly the case.

\section{Stability of the Finite-Volume Discretization with Implicit Time Marching Scheme}

For the implicit time marching scheme, as in the explicit discrete dispersion analysis, Figure 19 depicts $-\omega_{I}$ as a function of non-dimensional wavenumber $k$ using a $\Delta x$ of unity for various values of TSCF ranging from 100.0 to 0.1. As can be seen in Figure 19, all eigenvalues are in fact in the stable region (less than or equal to $-\omega_{I}=0$ ) whereas in Figure 18 many of the waves were unstable when using the explicit method. It is clear that a stable and accurate solution scheme can be constructed for the two-fluid MHD model using an implicit time marching procedure. It should be noted that the associated wavespeeds for the dispersion analysis of the discrete system with implicit time marching also approach the wavespeeds of the analytic dispersion analysis as $\Delta x$ approaches zero.

\section{Numerical Results for One-Dimensional Plasma Flows}

A first validation of the two-fluid MHD model is sought by considering its application to a well known one-dimensional problem. The initial value problem considered is the Brio-Wu test case. ${ }^{39}$ The implicit dual time stepping time marching scheme described in Section $\mathrm{V}$ was developed to generate the solutions described herein. The results from the implicit time marching scheme was compared with an explicit Hancock ${ }^{40}$ time marching scheme for accuracy. How TSCF and grid resolution affect the solution are also explored as well as the effect of collisions on the recovery of the equilibrium solution.

\section{A. Brio-Wu MHD Shock-Tube Problem}

The Brio-Wu test case is a MHD shock-tube problem that gives rise to some rather complex unsteady wave structure due to the interaction of the plasma with the electromagnetic fields. ${ }^{39}$ The ideal MHD solution to the Brio-Wu initial value problem is illustrated in Figure 20. The plasma waves that are present in the ideal MHD solution are, from left to right, a left moving fast rarefaction wave (FR), the slow compound (SC) wave, a contact discontinuity (CD), a slow shock (SS), and a right moving fast rarefaction wave (FR). 


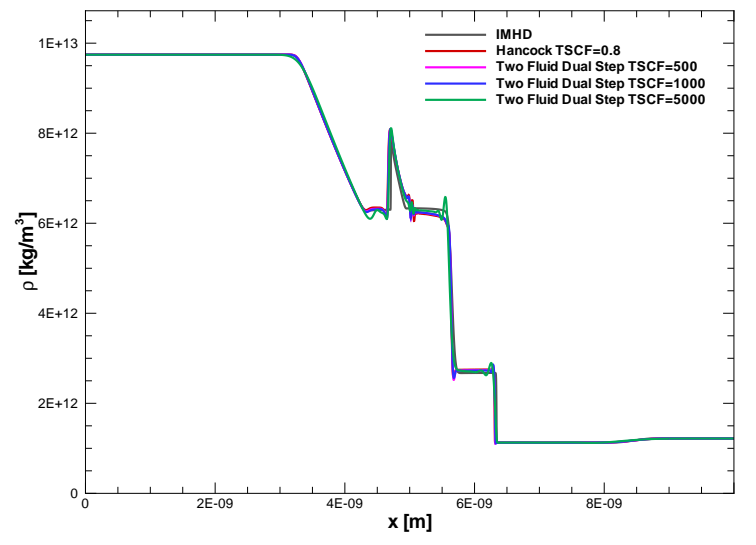

Figure 21. Density $\rho$ as a function of $x$ in the MHD limit.

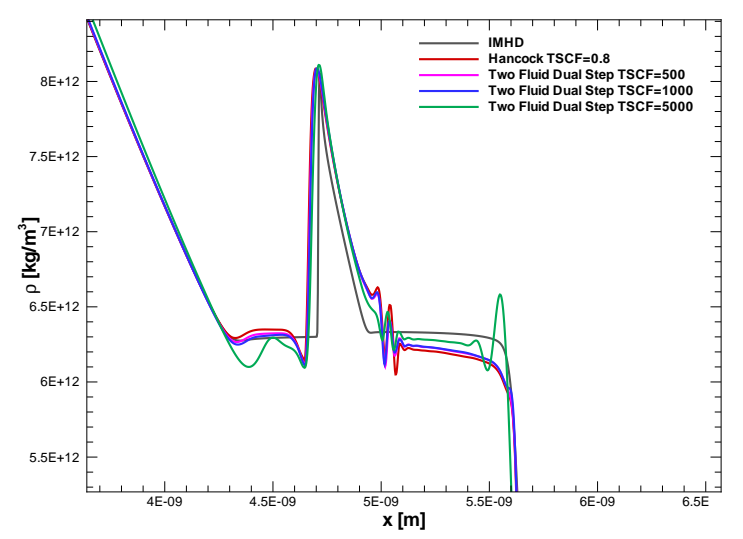

Figure 22. Close up of density $\rho$ as a function of $x$ for the compound shock in the MHD limit.

\section{B. Ideal MHD Limit}

To illustrate the recovery of the equilibrium ideal MHD limit by the two-fluid MHD model, the Brio-Wu initial value problem was considered with the following parameters:

$$
n_{0}=5.76 \times 10^{39} \mathrm{~m}^{-3}, \quad B_{0}=10^{10} \mathrm{~T}, \quad a_{\text {ref }}=3.0 \times 10^{6} \frac{\mathrm{m}}{\mathrm{s}}, \quad t=3.33 \times 10^{-16} \mathrm{~s} .
$$

A modified electron to ion mass ratio of 0.01 was adopted to improve the recovery of the equilibrium solution. Figure 21 shows the resulting density profile using an explicit Hancock scheme with a TSCF number of 0.8 and with 4000 cells along with profiles made with the implicit dual time stepping scheme developed above with a physical, outer, TSCF of 500, 1000 and 5000 with pseudo-time sub iteration TSCFs of 50000, 100000 and 500000 respectively. Figure 22 provides a close up of the compound shock in the ideal MHD limit. As can be seen in the figures, the two-fluid MHD model recovers the ideal MHD solution fairly well with only a few minor oscillations. Further, the explicit and dual time step implicit methods agree quite closely with each other for the physical TSCF of 500 and 1000 for the dual time step method. However, when the TSCF is increased further, there is a clear degradation in accuracy as $\Delta t$ becomes large, as well as a loss of solution monotonicity. We have a loss of monotonicity here due to the fact that monotonicity is not strictly enforced within the dual time stepping scheme.

There is a significant computational advantage to using the implicit dual time stepping method as illustrated in Table 1. As can be seen in the table, there is an order of magnitude increase in speed using the

Table 1. Computational time for solving the Brio-Wu test case using the explicit Hancock scheme and an implicit dual time stepping scheme

\begin{tabular}{|c|c|c|}
\hline Scheme & Physical TSCF & Time (minutes) \\
\hline Hancock & 0.8 & 1093 \\
Dual Step Implicit Euler & 500 & 154 \\
Dual Step Implicit Euler & 1000 & 80 \\
Dual Step Implicit Euler & 5000 & 52 \\
\hline
\end{tabular}

implicit dual time stepping scheme. Referring back to Figures 21 and 22, we can see that a physical TSCF of about 1000 provides a satisfactory increase in speed without degrading the quality of the solution for this Brio-Wu case.

\section{Comparison of Collisionless and Collisional Results}

To investigate the effects of interspecies collisions, the initial value problem of Brio and Wu was computed with the two-fluid MHD model and compared to the result of the two-fluid MHD model with no interspecies 


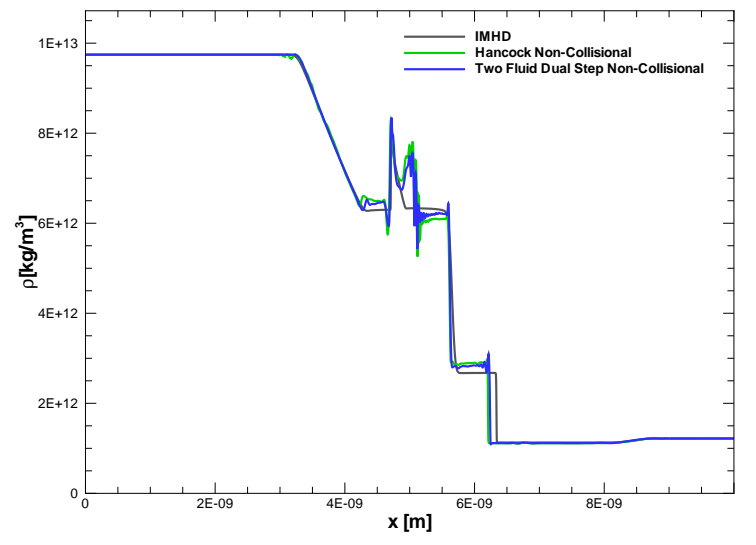

Figure 23. Density $\rho$ as a function of $x$ of the MHD limit of the two-fluid model comparing noncollisional flows solved with the explicit Hancock and implicit dual time stepping scheme.

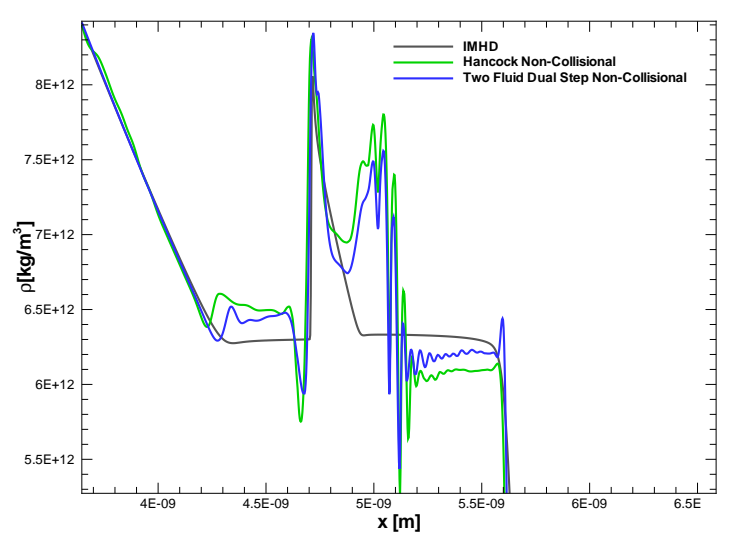

Figure 24. Close up density $\rho$ as a function of $x$ for the compound shock of the MHD limit comparing noncollisional flows solved with the explicit Hancock and implicit dual time stepping scheme.

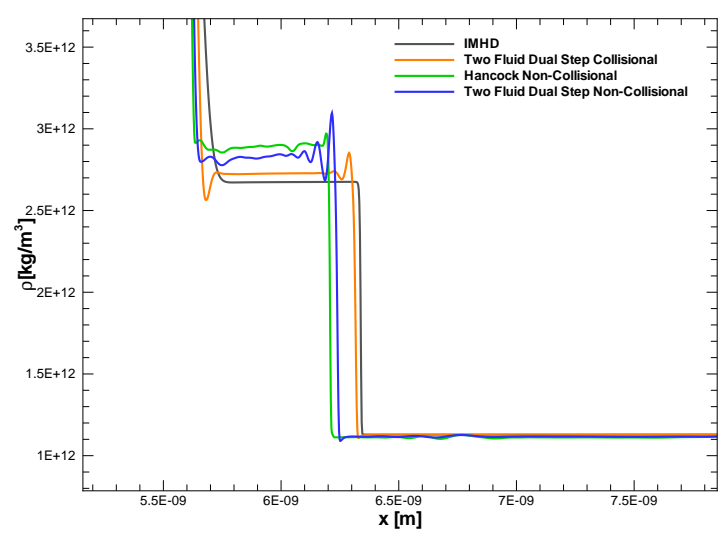

Figure 25. Close up density $\rho$ as a function of $x$ for the slow shock of the MHD limit comparing collisional and noncollisional flows solved with the implicit dual time stepping scheme.

collisions. Note that the two-fluid MHD 10-moment result with no interspecies collisions, but with self collisions, is equivalent to a 5-moment isotropic result with no interspecies collisions when there is sufficient self collisions to maintain an isotropic pressure. The interspecies collisionless result is expected to be similar to the results of Shumlak and Loverich. ${ }^{22}$

In Figure 23, the resulting density profiles of the ideal MHD and collisionless two-fluid MHD solutions are compared using 4000 cells, a physical TSCF of 1000 and with an electron/ion mass ratio of 0.01 . In order to obtain a stable solution, the TSCF of the explicit Hancock method was set to 0.1. It can be seen that the fully collisional solution presented before is much closer to the ideal MHD result, eliminating, or minimizing many of the oscillations that are present in the interspecies collisionless solution. The reduction of many of the oscillations can be seen by looking more carefully at the slow compound wave as seen in Figure 24 compared to the density profiles of the collisional result in Figure 22. Further, the slow shock is resolved more accurately in the fully collisional solution as illustrated in Figure 25. Evidently, interparticle collisions are essential in order to properly recover the ideal MHD limit. The computational cost of generating the collisionless solutions using the explicit Hancock and the implicit dual time stepping method for the two fluid MHD model were also measured and are included in Table 2. We are able to achieve a nearly 70 fold decrease in computational cost by using the implicit dual time step method of solving the two fluid MHD model. 
Table 2. Computational time for solving the non-collisional Brio-Wu test case using the explicit Hancock scheme and an implicit dual time stepping scheme

\begin{tabular}{|c|c|c|c|}
\hline Scheme & Physical TSCF & Number of Cells & Time (minutes) \\
\hline Hancock & 0.1 & 4000 & 6229 \\
Dual Step Implicit Euler & 1000 & 4000 & 93 \\
\hline
\end{tabular}

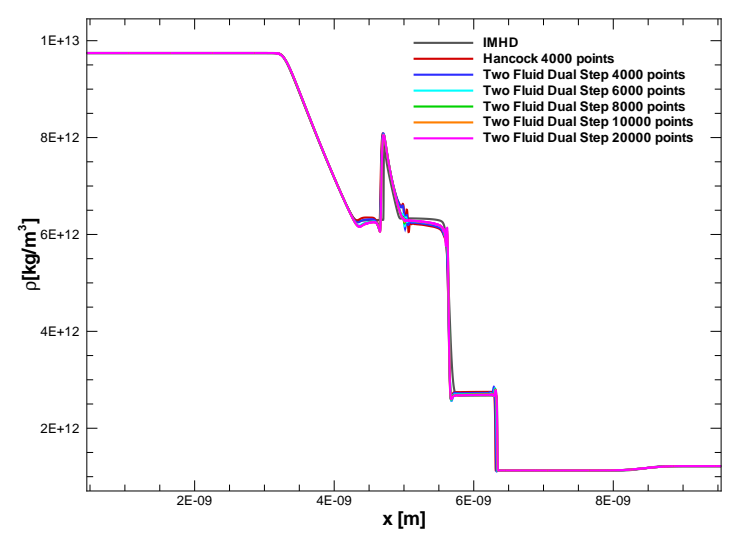

Figure 26. Density $\rho$ as a function of $x$ for the MHD limit of the two-fluid model for 4000,6000 , 8000,10000 , and 20000 cells solved with the implicit dual time stepping scheme.

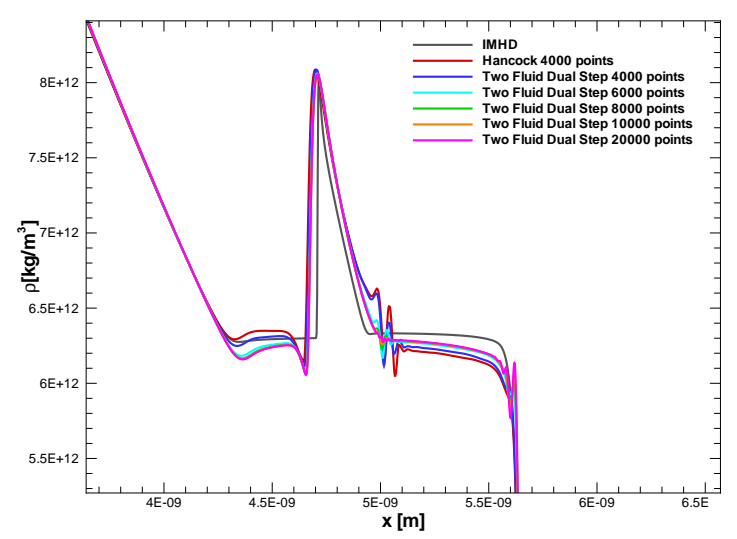

Figure 27. Close up density $\rho$ as a function of $x$ for the compound shock of the MHD limit 4000, $6000,8000,10000$ and 20000 cells solved with the implicit dual time stepping scheme.

\section{Grid Convergence}

The effects of mesh resolution on the solution of the Brio-Wu test case using the two-fluid MHD model and the implicit dual time step method were studied. The Brio-Wu test case was solved using 4000, 6000, 8000, 10000 and 20000 cells using the implicit dual time stepping scheme with a TSCF of 1000 and compared with the results of the Hancock explicit scheme for 4000 cells and a TSCF of 0.8, as well as the ideal MHD limit. The density profile results are presented in Figure 26 with a close up view of the slow compound wave in Figure 27.

The oscillations that appear in the 4000 cell explicit method are smaller in amplitude when solved using the dissipative implicit dual time step method with several of the waves disappearing at high grid resolutions. The wavelength of the oscillations also decrease. The wavelength attenuation is most dramatic when going from 4000 to 6000 cells, with a smaller change going from 6000 to 8000 cells and when moving from 8000 to 10000 cells, the wavelength remains largely the same. Therefore, the solution appears to converge at about 8000 cells. Unfortunately, an increase in grid resolution also causes new oscillations to appear and grow such as those in the contact discontinuity which can be seen on the right side of the slow compound wave density profile Figure 27. At 20000 cells we now have several more oscillations. Once again, we have a loss of monotonicity brought about by the large time steps, however this time, even though the time step taken by the implicit method defined by the TSCF remains the same, the time step is very large with respect to the cell size, as a characteristic wave can travel across many more cells in the same time step as $\Delta x$ decreases. Table 3 lists the computational time for each solution. As can be seen, even with a grid resolution of 20000 cells, the computational cost is still significantly less than the explicit method with only 4000 cells.

\section{E. Real Electron/Ion Mass Ratio}

We now relax one of the conditions for equilibrium convergence acceleration. The artificial mass ratio $m_{e} / m_{\text {ion }}=0.01$ is now dropped and the physical ratio $m_{e} / m_{\text {ion }}=1836$ is adopted in the Brio-Wu problem outlined above. Figure 28 compares the density profiles of the realistic Brio-Wu case using the explicit method with 4000 cells and a TSCF of 0.7 for stability, and several implicit dual time step profiles with a TSCF of 1000 and cell resolutions of 4000, 6000, 8000 and 10000 to illustrate spatial convergence with 
Table 3. Computational time for solving the Brio-Wu test case using the explicit Hancock scheme and an implicit dual time stepping scheme for various grid resolutions

\begin{tabular}{|c|c|c|c|}
\hline Scheme & Physical TSCF & Number of Cells & Time (minutes) \\
\hline Hancock & 0.8 & 4000 & 1093 \\
Dual Step Implicit Euler & 1000 & 4000 & 80 \\
Dual Step Implicit Euler & 1000 & 6000 & 152 \\
Dual Step Implicit Euler & 1000 & 8000 & 202 \\
Dual Step Implicit Euler & 1000 & 10000 & 267 \\
Dual Step Implicit Euler & 1000 & 20000 & 385 \\
\hline
\end{tabular}

a close up of the slow compound shock presented in Figure 29. Adopting a realistic electron/ion mass ratio has introduced several new oscillations in the solution since the system is now farther away from the equilibrium conditions required to produce a well defined Brio-Wu solution, mainly because of the faster electrons. Further, the problem is now less stable, and as a result, the explicit method must be run at a lower TSCF. The allowable time step is smaller as well due to an increase in the electron plasma frequency causing the computational cost to increase as illustrated in Table 4 which lists the computational time for the various solutions presented. Once again, the implicit dual time step method yields an order of magnitude decrease in computational cost over the explicit Hancock method. At higher grid resolutions made possible by the implicit dual time step method we can see that we can obtain a solution closer to the ideal MHD case.

Table 4. Computational time for solving the Brio-Wu test case using the explicit Hancock scheme and an implicit dual time stepping scheme for various grid resolutions and a physical electron/ion mass ratio

\begin{tabular}{|c|c|c|c|}
\hline Scheme & Physical TSCF & Number of Cells & Time (minutes) \\
\hline Hancock & 0.7 & 4000 & 4898 \\
Dual Step Implicit Euler & 1000 & 4000 & 323 \\
Dual Step Implicit Euler & 1000 & 6000 & 523 \\
Dual Step Implicit Euler & 1000 & 8000 & 660 \\
Dual Step Implicit Euler & 1000 & 10000 & 855 \\
\hline
\end{tabular}

\section{Conclusions}

A two-fluid MHD model based on the 10-moment Gaussian description, along with a full modelling of Maxwell's equations has been developed for a fully ionized plasma consisting of electrons and ions. The use of a 10-moment Gaussian closure allows for the modelling of large temperature and pressure anisotropies and the separate modelling of the species allows for temperature differences between the ions and electrons to be accurately modelled and resolved. A relaxation time approximation was used to model the collisional processes which coupled the plasmadynamics equations and Maxwell's equations. A dispersion analysis was undertaken in order to explore the physical behavior of the model. Due to the disparate nature of the time scales present in the model, it was shown that there is a wide range of temporal stiffness issues that must be addressed by any solution scheme procedure for the two-fluid MHD model.

A higher-order Godunov-type finite-volume upwind scheme solution procedure was proposed for solving the two-fluid MHD model and would use a HLLE Riemann flux function and Barth-Jesperson slope limiting. Discrete dispersion analysis revealed stability and stiffness issues with the proposed model. It was found that a fully implicit formulation of the numerical scheme would result in an unconditionally stable solution scheme and provide a basis for the solution of the two-fluid MHD model.

Numerical results have been presented for simplified one-dimensional initial value problems using both an explicit time marching scheme and an implicit dual time step scheme. This illustrated the potential for the model to simulate plasmas. It was shown that interparticle collisions are necessary in order to recover the 


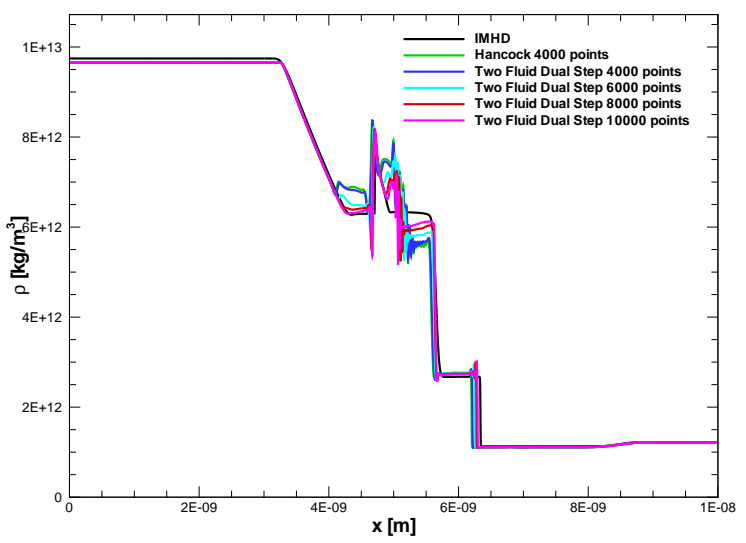

Figure 28. Density $\rho$ as a function of $x$ for the BrioWu test case with $m_{e} / m_{\text {ion }}=1 / 1836$ of the two-fluid model for $4000,6000,8000$, and 10000 cells solved with the implicit dual time stepping scheme.

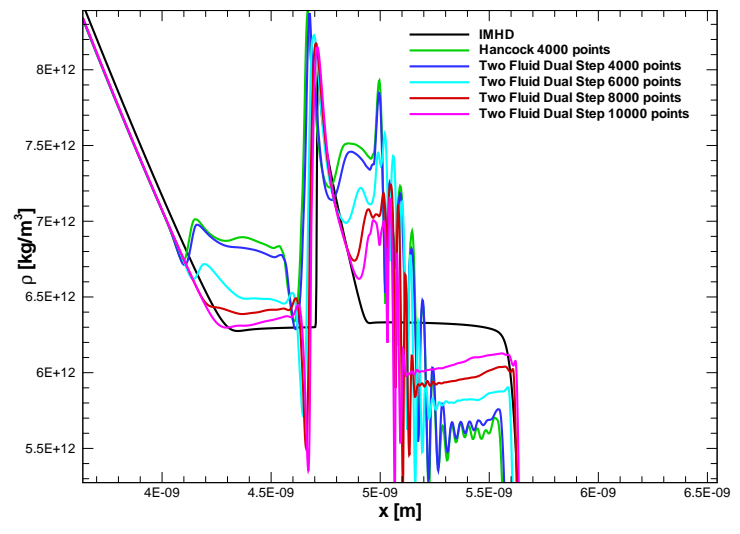

Figure 29. Close up density $\rho$ as a function of $x$ for the compound shock of the Brio-Wu test case with $m_{e} / m_{i o n}=1 / 1836$ of the two-fluid model for $4000,6000,8000$, and 10000 cells solved with the implicit dual time stepping scheme.

equilibrium MHD limit. Adopting the implicit dual time step scheme resulted in significant computational savings, producing, in some cases, smoother and higher resolution solutions at a fraction of the time required with the explicit scheme. It was illustrated that a stable and accurate implicit scheme could be created that can correctly recover the equilibrium limit.

Future work will concentrate on decreasing the computational costs further through preconditioning ${ }^{41}$ of the system of the discrete equations. High-order spatial and temporal schemes ${ }^{42,43}$ will also be considered in order to fully verify that converged equilibrium and non-equilibrium solutions can be obtained in one dimension. Extensions of the model are also planned for partially ionized and multi-dimensional plasma flows.

\section{Acknowledgements}

This research was supported by the Natural Sciences and Engineering Research Council of Canada (NSERC). The authors are very grateful to NSERC for this support. The first author would also like to thank NSERC for their generous support of his research though a Post Graduate Scholarship.

\section{References}

${ }^{1}$ Macheret, S. O., Shneider, M. N., and Miles, R. B., "Magnetohydrodynamic and Electrohydrodynamic Control of Hypersonic Flows of Weakly Ionized Plasmas," Vol. 42, No. 7, 2004, pp. 1378-1387.

${ }^{2}$ Seller, G., Capitelli, M., Longo, S., and Armenise, I., "Numerical MHD of Aircraft Re-Entry; Fluid Dynamic, Electromagnetic and Chemical Effects," Paper 2005-5048, AIAA, June 2003.

${ }^{3}$ Martinez-Sanchez, M. and Pollard, J. E., "Spacecraft Electric Propulsion - An Overview," Journal of Propulsion and Power, Vol. 14, No. 5, 1998, pp. 688-699.

${ }^{4}$ Brophy, J. R. and Noca, M., "Electric Propulsion for Solar System Exploration," Journal of Propulsion and Power, Vol. 14, No. 5, 1998, pp. 700-707.

${ }^{5}$ Krülle, G., Auweter-Kurtz, M., and Sasoh, A., "Technology and Application Aspects of Applied Field Magnetoplasmadynamic Propulsion," Journal of Propulsion and Power, Vol. 14, No. 5, 1998, pp. 754-763.

${ }^{6}$ Sovey, J. S., Rawlin, V. K., and Patterson, M. J., "A Synopsis of Ion Propulsion Development Projects in the United States: SERT I to Deep Space I," Paper 99-2270, AIAA, June 1999.

${ }^{7}$ Bond, T. A., Benson, G., Gallagher, J. T., and Matranga, M., "The NSTAR Ion Propulsion Subsystem for DS1," Paper 99-2972, AIAA, 1999.

${ }^{8}$ Racca, G. D. and Whitcomb, G. P., "The SMART-1 Mission," ESA Bulletin, August 1998.

${ }^{9}$ Oleson, S., "Electric Propulsion," Aerospace America, December 2002, pp. 58-59.

10 "ESA Achievements November 2001 - SMART-1," Document BR-200, November 2001.

${ }^{11}$ Auweter-Kurtz, M., Gölz, T., Habiger, H., Hammer, F., Kurtz, H., Riehle, M., and Sleziona, C., "High-Power Hydrogen Arcjet Thrusters," JPAP, Vol. 14, No. 5, Sept.-Oct. 1998.

${ }^{12}$ Díaz, F. R. C., "The VASIMR Rocket," Scientific American, , No. 2000-3756, July 2000. 
${ }^{13}$ Miura, K., Propellant Management System for a Microwave Thruster in a MOST class Spacecraft (BASc Thesis), Master's thesis, University of Toronto, 2001.

${ }^{14}$ Sutton, G. W. and Sherman, A., Engineering Magnetohydrodynamics, McGraw-Hill, Toronto, 1965.

${ }^{15}$ Chen, F. F., editor, Introduction to Plasma Physics, Plenum Press, New York, 1977.

${ }^{16}$ Gombosi, T. I., "Space Plasma Physics," Lecture notes.

${ }^{17}$ Groth, C. P. T., De Zeeuw, D. L., Gombosi, T. I., and Powell, K. G., "Global Three-Dimensional MHD Simulation of a Space Weather Event: CME Formation, Interplanetary Propagation, and and Interaction with the Magnetosphere," Journal of Geophysical Research, Vol. 105, No. A11, 2000, pp. 25,053-25,078.

${ }^{18}$ Groth, C. P. T., Zeeuw, D. L. D., Powell, K. G., Gombosi, T. I., and Stout, Q. F., "A Parallel Solution-Adaptive Scheme for Ideal Magnetohydrodynamics," Paper 99-3273, AIAA, June 1999.

${ }^{19}$ Ilin, A. V., Chang Díaz, F. R., Squire, J. P., Briezman, B. N., and Carter, M. D., "Particle Simulations of Plasma Heating in VASIMR," Paper 2000-3753, AIAA, July 2000.

${ }^{20}$ Bird, G. A., Molecular Gas Dynamics and the Direct Simulation of Gas Flows, Clarendon Press, Oxford, 1994.

${ }^{21}$ Breuer, K. S., Piekos, E. S., and Gonzales, D. A., "DSMC Simulations of Continuum Flows," Paper 95-2088, AIAA, June 1995.

${ }^{22}$ Shumlak, U. and Loverich, J., "Approximate Riemann solver for the two-fluid plasma model," Journal of Computational Physics, Vol. 187, 2003, pp. 620-638.

${ }^{23}$ Groth, C. P. T., "Numerical Modeling of Non-Equilibrium Micron-Scale Flows Using the Gaussian Moment Closure," Proceedings of the Eighth Annual Conference of the CFD Society of Canada, Montreal, Canada, June 11-13, 2000, Vol. 1, CFD Society of Canada, 2000, pp. 481-486.

${ }^{24}$ Brown, S. L., Roe, P. L., and Groth, C. P. T., "Numerical Solution of a 10-Moment Model for Nonequilibrium Gasdynamics," Paper 95-1677, AIAA, June 1995.

${ }^{25}$ Levermore, C. D., "Moment Closure Hierarchies for Kinetic Theories," Journal of Statistical Physics, Vol. 83, 1996, pp. 1021-1065.

${ }^{26}$ Levermore, C. D. and Morokoff, W. J., "The Gaussian Moment Closure for Gas Dynamics," SIAM Journal on Applied Mathematics, Vol. 59, No. 1, 1999, pp. 72-96.

${ }^{27} \mathrm{McD}$ onald, J. G. and Groth, C. P. T., "Numerical Modeling of Micron-Scale Flows Using the Gaussian Moment Closure," Paper 2005-5035, AIAA, June 2005.

${ }^{28}$ McDonald, J. G., Sachdev, J. S., and Groth, C. P. T., "Gaussian Moment Closure for the Modelling of Continuum and Micron-Scale Flows with Moving Boundaries," Paper, ICCFD, July 2006.

${ }^{29}$ Hansteen, V. H. and Leer, E., "Coronal Heating, Densities, and Temperatures and Solar Wind Acceleration," Journal of Geophysical Research, Vol. 100, No. A11, 1995, pp. 21577-21593.

${ }^{30}$ Bhatnagar, P. L., Gross, E. P., and Krook, M., "A Model for Collision Processes in Gases. I. Small Amplitude Processes in Charged and Neutral One-Component Systems," Physical Review, Vol. 94, No. 3, 1954, pp. 511-525.

${ }^{31}$ Gombosi, T. I., Gaskinetic Theory, Cambridge University Press, Cambridge, 1994.

${ }^{32}$ Miura, K., Development of an Extended Magnetohydrodynamic Model for Anisotropic Plasmas, Master's thesis, University of Toronto Institute for Aerospace Studies, 2004.

${ }^{33}$ Hittinger, J. A., Foundations for the Generalization of the Godunov Method to Hyperbolic Systems with Stiff Relaxation Source Terms, Ph.D. thesis, University of Michigan, 2000.

${ }^{34}$ Godunov, S. K., "Finite-Difference Method for Numerical Computations of Discontinuous Solutions of the Equations of Fluid Dynamics," Matematicheskii Sbornik, Vol. 47, 1959, pp. 271-306.

${ }^{35}$ Einfeldt, B., "On Godunov-Type Methods for Gas Dynamics," SIAM Journal on Numerical Analysis, Vol. 25, 1988, pp. 294-318.

${ }^{36}$ Barth, T. J., "Recent Developments in High Order K-Exact Reconstruction on Unstructured Meshes," Paper 93-0668, AIAA, January 1993.

${ }^{37}$ Venkateswaran, S. and Merkle, C. L., "Dual Time Stepping and Preconditioning for Unsteady Computations," Paper 95-0078, AIAA, January 1995.

${ }^{38}$ R. W. Hockney, J. W. E., Computer Simulation Using Particles, Institute of Physics Publishing, Bristol, 1988.

${ }^{39}$ Brio, M. and Wu, C. C., "An Upwind Differencing Scheme for the Equations of Ideal Magnetohydrodynamics," Journal of Computational Physics, Vol. 75, 1988, pp. 400-422.

${ }^{40}$ Suzuki, Y. and van Leer, B., "An extension of the upwind moment scheme to systems of 1-D nonlinear hyperbolicrelaxation equations," Proceedings of the 15th Annual Conference of the CFD Society of Canada, Toronto, Canada, May 27-31, 2007, CFD Society of Canada, 2007.

${ }^{41}$ Weiss, J. M. and Smith, W. A., "Preconditioning Applied to Variable and Constant Density Flows," AIAA Journal, Vol. 33, No. 11, 1995, pp. 2050-2057.

${ }^{42}$ Ivan, L. and Groth, C., "High-Order Central ENO Finite-Volume Scheme with Adaptive Mesh Refinement," Paper 07-4323, AIAA, June 2007.

${ }^{43}$ Bijl, H., Carpenter, M. H., Vatsa, V. N., and Kennedy, C. A., "Implicit Time Integration Schemes for the Unsteady Compressible Navier-Stokes Equations: Laminar Flow," Journal of Computational Physics, Vol. 179, 2002, pp. 313-329. 